Copyright (C) 2014 IEEE. Personal use of this material is permitted. Permission from IEEE must be obtained for all other uses, in any current or future media, including reprinting/republishing this material for advertising or promotional purposes, creating new collective works, for resale or redistribution to servers or lists, or reuse of any copyrighted component of this work in other works. 


\title{
On MAC-BC Duality of Multihop MIMO Relay Channel with Imperfect Channel Knowledge
}

\author{
Ali Cagatay Cirik, Yue Rong, Senior Member, IEEE, Yiming Ma, and Yingbo Hua, Fellow, IEEE
}

\begin{abstract}
In this paper, we establish the signal-to-interferencenoise ratio (SINR) duality between multiple access (MAC) and broadcast (BC) multihop amplify-and-forward (AF) multipleinput multiple-output (MIMO) relay systems under an imperfect channel state model, which is a generalization of several previously established MAC-BC duality results. We show that identical SINRs in the MAC and BC systems can be achieved by two approaches. The first one is to use the Hermitian transposed MAC relay amplifying matrices at the relay nodes in the $\mathrm{BC}$ system, under the same total network transmission power constraint. The second one is to use the scaled and Hermitian transposed MAC relay amplifying matrices in the BC system, under the transmission power constraint at each node of the system, where the scaling factors are obtained by swapping the power constraints of the nodes in the MAC system. Moreover, we derive the MAC-BC mean-squared error (MSE) and achievable sum-rate (or mutual information (MI)) duality properties based on the SINR duality. Numerical results show the utility of the duality results established.
\end{abstract}

\section{INTRODUCTION}

Multiple-input multiple-output (MIMO) relays are important for wireless communication networks because they can be used to reduce the path loss, increase the power efficiency, and improve the network coverage. MIMO relays can be used for multiple access (MAC) - from multiple users to a base station. They can also be used for broadcast (BC) - from a base station to multiple users. In this paper, we consider a chain of multihop amplify-and-forward (AF) MIMO relays for either MAC or BC. To achieve a desired performance for such a system, the transformation matrices applied at the source, the destination, and the relays need to be chosen properly. Our contribution in this paper is about MAC-BC duality properties of the multihop MIMO relay system in terms of these transformation matrices under an imperfect channel state information (CSI) model. Due to the multihop topology and imperfect CSI assumption, the achievable rate is a complicated function of the source, relay and receiver matrices, which makes both the proof of duality and the optimization problems associated with multihop MIMO relay networks much more challenging than the existing works with simpler network topology and/or perfect CSI assumption. In other words, a rigorous proof of the MAC-BC duality of multihop MIMO

A. C. Cirik, Y. Ma, and Y. Hua are with the Department of Electrical Engineering, University of California, Riverside, CA 92521, USA (email: acirik@ee.ucr.edu,yma@ee.ucr.edu, and yhua@ee.ucr.edu).

Y. Rong is with the Department of Electrical and Computer Engineering, Curtin University of Technology, Bentley, WA 6102, Australia (email: y.rong@curtin.edu.au).

The work of Y. Rong was supported in part by the Australian Research Councils Discovery Projects funding scheme (project number DP140102131). relay channel with imperfect channel knowledge is much more complex than that of the existing MAC-BC duality results.

\section{A. Literature Review of Existing MAC-BC Duality Properties}

To the best of our knowledge, MAC-BC duality results available in the literature can be divided into the following three main categories (also summarized in Table I).

1) SINR Duality: The MAC-BC SINR duality for singlehop multiple-input single-output (MISO) systems was derived independently in [1] and [2]. Based on this SINR duality, sum-power minimization problem subject to minimum SINR requirements and the SINR balancing problem were solved in [2]. The SINR duality result for MISO systems was extended to MIMO systems with multi-antenna receivers/transmitters in [3] and [4]. Recently, the MAC-BC SINR duality for single-hop MIMO systems has been extended to two-hop amplify-and-forward (AF) MIMO relay systems, where all nodes in the system are equipped with multiple antennas [5]. It is shown in [5] that in a MAC system, SINRs identical to that of the BC system can be achieved by employing a scaled Hermitian transpose of the relay amplifying matrix used in the BC system, and the scaling factor is obtained by swapping the transmission power constraints at the source node and the relay node. This result generalizes the SINR duality established for single-hop MIMO systems in [1]-[4]. The authors of [6] extended the two-hop MAC-BC SINR duality results in [5] to multihop AF MIMO relay systems with any number of hops and any number of antennas at each node. Recently, a generalized multihop MIMO AF relay network, which has multiple sources, multiple destinations and multiple relays is considered in [7]. Dualities for this network under single network linear constraint and per-hop linear constraint are established.

The aforementioned SINR duality results are established by assuming that the exact CSI is available in the system. However, in practical communication systems, the CSI knowledge is obtained through channel training/estimation. Due to limited length of training sequences, channel noise, quantization errors, outdated channel estimates, and/or time-varying nature of wireless channels, there is mismatch between the estimation and the exact CSI, which may substantially degrade the system performance. Therefore, robust precoder/receive filter design that explicitly takes into account the uncertainties in the channel model should be considered in practical applications. This motivates the authors of [8] to establish the SINR duality under imperfect CSI for single-hop MISO systems, which generalizes the SINR duality with perfect CSI in [1] and [2]. 
TABLE I

EXISTING MAC-BC DUALITY RESUlts

\begin{tabular}{|c|c|c|c|c|c|c|c|}
\hline & & \multicolumn{5}{|c|}{ MISO } & \multicolumn{3}{c|}{ MIMO } \\
\hline & & SH & TH & MH & SH & TH & MH \\
\hline \multirow{3}{*}{ PCSI } & SINR & {$[1],[2]$} & {$[5]$} & {$[6],[7]$} & {$[3],[4]$} & {$[5]$} & {$[6],[7]$} \\
& MSE & {$[9]$} & {$[17]$} & this paper & {$[4],[10],[11]$} & {$[17]$} & this paper \\
& Capacity/MI & {$[18] /[1],[2]$} & NA/[5], [21] & NA/[6]-[7], [21] & {$[19]-[20] /[3]-[4]$} & NA/[5] & NA/[6]-[7] \\
\hline \multirow{3}{*}{ ICSI } & SINR & {$[8]$} & this paper & this paper & this paper & this paper & this paper \\
& MSE & {$[8],[12]$} & {$[17]$} & this paper & {$[13]-[16]$} & {$[17]$} & this paper \\
& Capacity/MI & NA/[8] & NA/this paper & NA/this paper & NA/this paper & NA/this paper & NA/this paper \\
\hline
\end{tabular}

PCSI: Perfect channel state information, ICSI: Imperfect channel state information

MIMO: Multi-input multi-output, MISO: Multi-input single-output

SH: Single-hop, TH: Two-hop, MH: Multihop

NA: Not Available

2) MSE Duality: The MAC-BC MSE duality was first derived for MISO systems with a sum power constraint in [9] and then extended to MIMO systems in [4], [10], and [11]. It was observed in [10] that under a total power constraint, any MSE point that is achievable in the MAC system can also be attained in the BC system.

The MSE duality results obtained in [4] and [9]-[11] are based on the assumption that the exact CSI is available in the system. For systems with imperfect CSI, the MSE duality has been established in [8] and [12] for single-hop MISO systems, and in [13] for single-hop MIMO systems. However, channel correlation among antenna elements is not considered in [8], [12], and [13]. The sum-MSE duality (based on the Karush-Kuhn-Tucker conditions associated with the optimization problems in the MAC and BC systems) and three kinds of MSE duality (individual stream MSE, individual user MSE, sum-MSE) have been established in [14] and [15], respectively for single-hop MIMO systems with imperfect CSI and antenna correlation only at the base station. The MSE duality results in [15] is extended in [16] by considering the antenna correlation at both the base station and the users. In fact, the duality results obtained in [16] can be viewed as the extension of those in [11] to the imperfect CSI case. Recently, the sum-MSE MAC-BC duality in single-hop MIMO systems under imperfect CSI has been extended to two-hop AF MIMO relay systems in [17].

3) Capacity Duality: The MAC-BC rate-region duality for single antenna terminals or single stream transmission with multi-antenna terminals can be readily derived from the SINR duality. In particular, the sum capacity duality was proven for MISO systems in [1] by showing that the achievable sum-rate with Costa precoding in the $\mathrm{BC}$ system is the same as the maximum sum-rate in the MAC system. The rate regions of the MAC and the BC under Gaussian signaling and nonlinear interference cancellation have been proven to be the same, for the single-antenna case [18], for the MIMO case supporting an arbitrary number of data streams per user [19], and for the overlap of the dirty-paper coding rate region and the capacity region [20]. The authors of [21] derived the rate-region duality for multihop AF MIMO relay systems with single antenna source and destination nodes, which generalizes the MAC-BC rate-region duality results of [1] and [19].

\section{B. Contributions of Our Work}

To the best of our knowledge, so far no work has been done to prove the SINR (MSE, MI) duality for multihop AF MIMO relay systems that consider imperfect CSI and antenna correlation at all nodes (see Table I). In this paper, we consider that the antennas of all the nodes in the system exhibit spatial correlations and the CSI at each hop is imperfect. Due to the coupling between multiple system parameters (source and relay precoding matrices and receiver matrices) and the mismatch between the exact and estimated CSI, a rigorous proof of the duality results is much more complex than that of the existing MAC-BC duality results. We show that under imperfect CSI, stream-wise identical SINR (MSE, MI) can be achieved in multihop AF MIMO MAC and BC relay systems through two approaches. First, if there is only total network transmission power constraint and no power constraint at individual nodes, then duality can be achieved by employing $\mathbf{F}_{l}$ and $\mathbf{F}_{L-l}^{H}, l=1, \ldots, L-1$, as the relay amplifying matrices at the $l$ th relay node of the BC and the MAC MIMO relay systems, respectively, where $L$ is the number of hops of the relay network. Second, with transmission power constraint at each node of the relay network, duality can be established when $c_{l} \mathbf{F}_{l}$ and $\mathbf{F}_{L-l}^{H}, l=1, \ldots, L-1$, are employed as the amplifying matrices at the $l$ th relay node of the $\mathrm{BC}$ and the MAC relay systems, respectively. In this case, the scaling factor $c_{l}>0$ is obtained by swapping the power constraints at the $l$ th node of the BC system and the $(L+1-l)$-th node of the MAC system, $l=1, \ldots, L{ }^{1}$

Furthermore, we prove that the two approaches developed above are not only valid for MIMO relay systems with linear transceivers at the source and the destination nodes, but also hold if a receiver employing successive interference cancellation (SIC) is used at the destination of the MAC MIMO relay

\footnotetext{
${ }^{1}$ There is no loss of generality in assuming $c_{l}$ to be a non-negative real number [5].
} 
system, and a transmitter employing dirty paper coding (DPC) is used at the source node of the BC MIMO relay channel. As an application of this MAC-BC duality, the complicated robust multihop MIMO BC system design problem under imperfect CSI can be efficiently solved by focusing on an equivalent multihop MIMO MAC problem.

As an application of the duality results established, we consider the design of source precoding matrix and relay amplifying matrices for multihop AF-MIMO relay systems under sum-MSE optimization problem. Simulation results demonstrate that both the MAC and $\mathrm{BC}$ systems achieve the same sum-MSE and bit error rate (BER).

In this paper, we define the SINR as an estimated SINR when only an estimate of the channel response is available. An estimated SINR is a ratio of signal power coupled with the estimated channel over interference-plus-noise power plus the channel estimation error variance coupled with the signal power. Such a definition of SINR is consistent with those defined in prior works [22]-[25]. This estimated SINR is more meaningful than an exact SINR when the channel knowledge at a receiver is not exact. In fact, when there is only an estimated channel response, the performance of the receiver is directly governed by the estimated SINR instead of the exact SINR, in the sense that the channel estimation error affects the effective SINR as an additional noise.

The following notations are used in this paper. Matrices and vectors are denoted as bold capital and lowercase letters, respectively. For matrices, (.) $)^{T}$ and $(.)^{H}$ denote transpose and conjugate transpose, respectively. $\mathbb{E}[$.$] stands for the statistical$ expectation; $\mathbf{I}_{N}$ denotes an $N \times N$ identity matrix; $\operatorname{tr}\{$. stands for matrix trace; $\operatorname{diag}\left\{a_{1}, \cdots, a_{n}\right\}$ denotes a diagonal matrix with the diagonal elements given by $a_{1}, \cdots, a_{n}$, and $\|.\|_{2}$ stands for the vector Euclidean norm. For matrices $\mathbf{A}_{i}, \bigotimes_{i=l}^{k}\left(\mathbf{A}_{i}\right) \triangleq \mathbf{A}_{l} \ldots \mathbf{A}_{k}$. For example, $\bigotimes_{i=1}^{3}\left(\mathbf{A}_{i}\right) \triangleq$ $\mathbf{A}_{1} \mathbf{A}_{2} \mathbf{A}_{3}$ and $\bigotimes_{i=3}^{1}\left(\mathbf{A}_{i}\right) \triangleq \mathbf{A}_{3} \mathbf{A}_{2} \mathbf{A}_{1}$. bd(.) stands for a block diagonal matrix, and $\Re\{$.$\} denotes the real part.$

\section{SYSTEM MODEL}

We consider a wireless communication system with one base station (BS), $L-1(L \geq 2)$ relay nodes and $K$ user nodes, where the BS is equipped with $N_{1}$ antennas and the $(l-1)$ th relay node is equipped with $N_{l}$ antennas, $l=2, \ldots, L$. The $i$ th user, $i=1, \ldots, K$, transmits (receives) $N_{b}^{(i)}$ data streams using $N_{L+1}^{(i)}$ antennas in the MAC (BC) system. We denote $N_{b}=\sum_{i=1}^{K} N_{b}^{(i)}$ as the total number of independent data streams from all users and $N_{L+1}=\sum_{i=1}^{K} N_{L+1}^{(i)}$ as the total number of antennas of all users. In order to support $N_{b}$ data streams simultaneously, $N_{b} \leq \min \left(N_{1}, N_{2}, \ldots, N_{L+1}\right)$ should be satisfied. However, if a nonlinear transmitter is installed at the source node or a nonlinear receiver is installed at the destination node of a MIMO relay system, $N_{b}$ can be greater than $\min \left(N_{1}, N_{2}, \ldots, N_{L+1}\right)$ [6]. We assume the orthogonality among different hops, also adopted in [5]-[7], [21], meaning that the signal transmitted by the $l$ th node can only be received by the $(l+1)$-th node due to the propagation pathloss and proper channel reuse. Thus, there are $L$ hops between the source and destination nodes. Each relay node works in half-duplex mode and employs a linear AF (non-regenerative) relay matrix to amplify and forward its received signals.

\section{A. Multihop BC MIMO Relay System}

In the multihop BC MIMO relay system shown in Fig. 1, the source symbol vector $\mathbf{s}_{i}^{B}=\left[s_{i, 1}^{B}, s_{i, 2}^{B}, \ldots, s_{i, N_{b}^{(i)}}^{B}\right]^{T}$ of size $N_{b}^{(i)} \times 1$ from the $i$ th user is linearly precoded by matrix $\mathbf{U}_{i} \mathbf{Q}_{i}^{\frac{1}{2}} \in \mathcal{C}^{N_{1} \times N_{b}^{(i)}}$, where $\mathbf{U}_{i}=\left[\mathbf{u}_{i}^{(1)}, \mathbf{u}_{i}^{(2)}, \ldots, \mathbf{u}_{i}^{\left(N_{b}^{(i)}\right)}\right]$ with $\left\|\mathbf{u}_{i}^{(j)}\right\|_{2}=1$ and $\mathbf{Q}_{i}=\operatorname{diag}\left\{q_{i}^{(1)}, q_{i}^{(2)}, \ldots, q_{i}^{\left(N_{b}^{(i)}\right)}\right\}$ with $q_{i}^{(j)}, j=1, \ldots, N_{b}^{(i)}, i=1, \ldots, K$, being the power allocated to the $j$ th data stream of the $i$ th user. We assume that user symbols are independent and have unit-power, i.e., $\mathbb{E}\left[\mathbf{s}_{i}^{B}\left(\mathbf{s}_{i}^{B}\right)^{H}\right]=\mathbf{I}_{N_{b}^{(i)}}$. The BS transmits the $N_{1} \times 1$ linearly precoded symbol vector $\mathbf{x}_{1}^{B}=\sum_{i=1}^{K} \mathbf{U}_{i} \mathbf{Q}_{i}^{\frac{1}{2}} \mathbf{s}_{i}^{B}=\mathbf{U Q}^{\frac{1}{2}} \mathbf{S}^{B}$, where $\mathbf{U}=\left[\mathbf{U}_{1}, \mathbf{U}_{2}, \ldots, \mathbf{U}_{K}\right], \mathbf{Q}=\operatorname{bd}\left(\mathbf{Q}_{1}, \mathbf{Q}_{2}, \ldots, \mathbf{Q}_{K}\right)$, and $\mathbf{s}^{B}=\left[\left(\mathbf{s}_{1}^{B}\right)^{T},\left(\mathbf{s}_{2}^{B}\right)^{T}, \ldots,\left(\mathbf{s}_{K}^{B}\right)^{T}\right]^{T}$. The $N_{l} \times 1$ received signal vector at the $(l-1)$-th relay node of the BC system can be written as

$$
\mathbf{y}_{l}^{B}=\mathbf{H}_{l-1} \mathbf{x}_{l-1}^{B}+\mathbf{n}_{l}, \quad l=2, \ldots, L
$$

where $\mathbf{H}_{l} \in \mathcal{C}^{N_{l+1} \times N_{l}}, l=1, \ldots, L-1$, is the MIMO channel matrix between the $(l+1)$-th and the $l$ th node, $\mathbf{x}_{l-1}^{B} \in \mathcal{C}^{N_{l-1} \times 1}$ is the signal vector transmitted by the $(l-1)$ th node, $l=2, \ldots, L+1, \mathbf{n}_{l} \in \mathcal{C}^{N_{l} \times 1}$ is the independent and identically distributed (i.i.d.) additive white Gaussian noise (AWGN) vector at the $(l-1)$-th relay node, $l=2, \ldots, L$. We assume that all noises are complex circularly symmetric with zero mean and unit variance. The transmitted signal vector at the $(l-1)$-th relay node is written as

$$
\mathbf{x}_{l}^{B}=c_{l-1} \mathbf{F}_{l-1} \mathbf{y}_{l}^{B}, \quad l=2, \ldots, L
$$

where $c_{l-1} \mathbf{F}_{l-1} \in \mathcal{C}^{N_{l} \times N_{l}}$ is the amplifying matrix at the $(l-1)$-th relay node, and $c_{l}>0$ is a scaling coefficient which is important for studying the MAC-BC duality [21]. Using (1) and (2), the received signal vector at the first and $l$ th relay node is written, respectively as

$$
\begin{aligned}
\mathbf{y}_{2}^{B} & =\mathbf{H}_{1} \mathbf{U Q}^{\frac{1}{2}} \mathbf{s}^{B}+\mathbf{n}_{2}, \\
\mathbf{y}_{l+1}^{B} & =\mathbf{H}_{l} \bigotimes_{m=l-1}^{1}\left(c_{m} \mathbf{F}_{m} \mathbf{H}_{m}\right) \mathbf{U Q}^{\frac{1}{2}} \mathbf{s}^{B}+\mathbf{n}_{l+1} \\
& +\sum_{k=2}^{l} \bigotimes_{m=l}^{k}\left(c_{m-1} \mathbf{H}_{m} \mathbf{F}_{m-1}\right) \mathbf{n}_{k}, \quad l=2, \ldots, L-1 .
\end{aligned}
$$

The received signal vector at the $i$ th user node is given by

$$
\mathbf{y}_{L+1}^{(i)^{B}}=\mathbf{H}_{L_{i}} c_{L-1} \mathbf{F}_{L-1} \mathbf{y}_{L}^{B}+\mathbf{n}_{L+1}^{(i)}, \quad i=1, \cdots, K
$$

where $\mathbf{H}_{L_{i}} \in \mathcal{C}^{N_{L+1}^{(i)} \times N_{L}}$ is the MIMO channel matrix between the $i$ th user node and the $(L-1)$-th relay node and $\mathbf{n}_{L+1}^{(i)} \in \mathcal{C}^{N_{L+1}^{(i)} \times 1}$ is the i.i.d. AWGN vector at the $i$ th user node. 


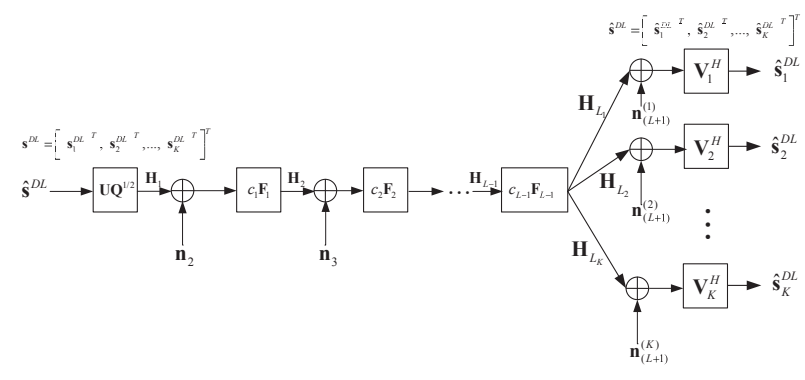

Fig. 1. Multihop BC AF MIMO Relay System

A linear receiver matrix $\mathbf{V}_{i} \in \mathcal{C}^{N_{L+1}^{(i)} \times N_{b}^{(i)}}$ is applied at the $i$ th user node to estimate the symbol vector $\mathbf{s}_{i}^{B}$, where the columns of $\mathbf{V}_{i}$ are assumed to satisfy $\left\|\mathbf{v}_{i}^{(j)}\right\|_{2}=1, j=$ $1, \ldots, N_{b}^{(i)}, i=1, \ldots, K$. The estimated symbol vector $\hat{\mathbf{s}}_{i}^{B}$ is expressed as

$$
\hat{\mathbf{s}}_{i}^{B}=\mathbf{V}_{i}^{H} \mathbf{y}_{L+1}^{(i)^{B}} .
$$

Using (3)-(6), we have the decision variable of the $j$ th data symbol of the $i$ th user as

$$
\begin{aligned}
& \hat{s}_{i, j}^{B}=\underbrace{\left(\mathbf{v}_{i}^{(j)}\right)^{H} \mathbf{H}_{L_{i}} \bigotimes_{m=L-1}^{1}\left(c_{m} \mathbf{F}_{m} \mathbf{H}_{m}\right) \mathbf{u}_{i}^{(j)}\left(q_{i}^{(j)}\right)^{\frac{1}{2}} s_{i, j}^{B}}_{\text {desired signal }} \\
& +\underbrace{\left(\mathbf{v}_{i}^{(j)}\right)^{H} \mathbf{H}_{L_{i}} \sum_{l=1, l \neq j}^{N_{b}^{(i)}} \bigotimes_{m=L-1}^{1}\left(c_{m} \mathbf{F}_{m} \mathbf{H}_{m}\right) \mathbf{u}_{i}^{(l)}\left(q_{i}^{(l)}\right)^{\frac{1}{2}} s_{i, l}^{B}}_{\text {intra-user interference }} \\
& +\underbrace{\left(\mathbf{v}_{i}^{(j)}\right)^{H} \mathbf{H}_{L_{i}} \sum_{k=1, k \neq i}^{K} \sum_{l=1}^{N_{b}^{(k)}} \bigotimes_{m=L-1}^{1}\left(c_{m} \mathbf{F}_{m} \mathbf{H}_{m}\right) \mathbf{u}_{k}^{(l)}\left(q_{k}^{(l)}\right)^{\frac{1}{2}} s_{k, l}^{B}}_{\text {noise propagated from previous hops }} \\
& +\underbrace{\left(\mathbf{v}_{i}^{(j)}\right)^{H} c_{L-1} \mathbf{H}_{L_{i}} \mathbf{F}_{L-1}\left(\sum_{k=2}^{L-1} \bigotimes_{m=L-1}^{k}\left(c_{m-1} \mathbf{H}_{m} \mathbf{F}_{m-1}\right) \mathbf{n}_{k}+\mathbf{n}_{L}\right)}_{j=1, \ldots, N_{b}^{(i)}, \quad i=1, \ldots, K . \quad(7)} \\
& +\underbrace{\left(\mathbf{v}_{i}^{(j)}\right)^{H} \mathbf{n}_{L+1}^{(i)}}_{\text {noise at the destination }}, \quad
\end{aligned}
$$

\section{B. Multihop MAC MIMO Relay System}

For the multihop MAC MIMO relay system shown in Fig. 2, the roles of the BS and user nodes at the BC MIMO relay system are swapped. The Hermitian transpose of the channel matrices used in the BC system are employed in the MAC system. The $i$ th user node linearly precodes the symbol vector $\mathbf{s}_{i}^{M}=\left[s_{i, 1}^{M}, s_{i, 2}^{M}, \ldots, s_{i, N_{b}^{(i)}}^{M}\right]^{T}$ using the matrix $\mathbf{V}_{i} \mathbf{P}_{i}^{\frac{1}{2}}$, where $\mathbf{P}_{i}=\operatorname{diag}\left\{p_{i}^{(1)}, p_{i}^{(2)}, \ldots, p_{i}^{\left(N_{b}^{(i)}\right)}\right\}$ with $p_{i}^{(j)}$, $j=1, \ldots, N_{b}^{(i)}, i=1, \ldots, K$, being the power allocated to the $j$ th data stream of the $i$ th user. The $l$ th node, i.e. $(l-1)$ th relay node, $l=2, \ldots, L$, employs $\mathbf{F}_{L+1-l}^{H}$ to amplify and forward received signals. The received signal vector at the first

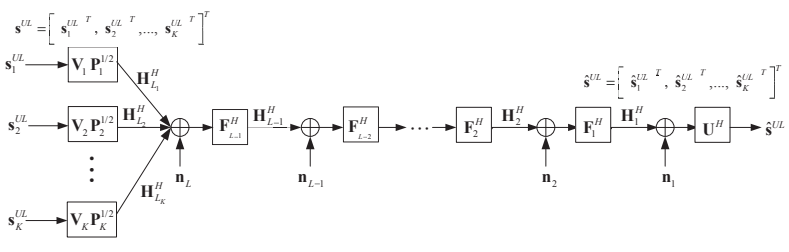

Fig. 2. Multihop MAC AF MIMO Relay System

and the $(L+1-l)$-th receiving node of the MAC system is given, respectively, by

$$
\begin{aligned}
\mathbf{y}_{2}^{M} & =\mathbf{H}_{L}^{H} \mathbf{V} \mathbf{P}^{\frac{1}{2}} \mathbf{s}^{M}+\mathbf{n}_{L} \\
\mathbf{y}_{L+2-l}^{M} & =\bigotimes_{m=l}^{L-1}\left(\mathbf{H}_{m}^{H} \mathbf{F}_{m}^{H}\right) \mathbf{H}_{L}^{H} \mathbf{V} \mathbf{P}^{\frac{1}{2}} \mathbf{s}^{M}+\mathbf{n}_{l} \\
& +\sum_{k=l}^{L-1} \bigotimes_{m=l}^{k}\left(\mathbf{H}_{m}^{H} \mathbf{F}_{m}^{H}\right) \mathbf{n}_{k+1}, \quad l=1, \ldots, L-1 .
\end{aligned}
$$

Here, we defined $\mathbf{s}^{M}=\left[\left(\mathbf{s}_{1}^{M}\right)^{T},\left(\mathbf{s}_{2}^{M}\right)^{T}, \ldots,\left(\mathbf{s}_{K}^{M}\right)^{T}\right]^{T}$, $\mathbf{V}=\operatorname{bd}\left(\mathbf{V}_{1}, \mathbf{V}_{2}, \ldots, \mathbf{V}_{K}\right), \mathbf{P}=\operatorname{bd}\left(\mathbf{P}_{1}, \mathbf{P}_{2}, \ldots, \mathbf{P}_{K}\right)$, and $\mathbf{H}_{L}^{H}=\left[\mathbf{H}_{L_{1}}^{H}, \mathbf{H}_{L_{2}}^{H} \ldots, \mathbf{H}_{L_{K}}^{H}\right]$. A linear receiver matrix $\mathbf{U}_{i}$ is used at the BS to estimate the transmitted symbol vector of user $i$, and the estimated symbol vector $\hat{\mathbf{s}}_{i}^{M}$ is expressed as

$$
\hat{\mathbf{s}}_{i}^{M}=\mathbf{U}_{i}^{H} \mathbf{y}_{L+1}^{M} .
$$

Using (8)-(10), we have the decision variable of the $j$ th data symbol of the $i$ th user as

$$
\begin{aligned}
& \hat{s}_{i, j}^{M}=\underbrace{\left(\mathbf{u}_{i}^{(j)}\right)^{H} \bigotimes_{m=1}^{L-1}\left(\mathbf{H}_{m}^{H} \mathbf{F}_{m}^{H}\right) \mathbf{H}_{L_{i}}^{H} \mathbf{v}_{i}^{(j)}\left(p_{i}^{(j)}\right)^{\frac{1}{2}} s_{i, j}^{M}}_{\text {desired signal }} \\
& +\underbrace{\left(\mathbf{u}_{i}^{(j)}\right)^{H} \sum_{l=1, l \neq j}^{N_{b}^{(i)}} \bigotimes_{m=1}^{L-1}\left(\mathbf{H}_{m}^{H} \mathbf{F}_{m}^{H}\right) \mathbf{H}_{L_{i}}^{H} \mathbf{v}_{i}^{(l)}\left(p_{i}^{(l)}\right)^{\frac{1}{2}} s_{i, l}^{M}}_{\text {intra-user interference }} \\
& +\underbrace{\left(\mathbf{u}_{i}^{(j)}\right)^{H} \sum_{k=1, k \neq i}^{K} \sum_{l=1}^{N_{b}^{(k)}} \bigotimes_{m=1}^{L-1}\left(\mathbf{H}_{m}^{H} \mathbf{F}_{m}^{H}\right) \mathbf{H}_{L_{k}}^{H} \mathbf{v}_{k}^{(l)}\left(p_{k}^{(l)}\right)^{\frac{1}{2}} s_{k, l}^{M}}_{\text {noise propagated from previous hops }} \\
& +\underbrace{\left(\mathbf{u}_{i}^{(j)}\right)^{H} \sum_{k=1}^{L-1} \bigotimes_{m=1}^{k}\left(\mathbf{H}_{m}^{H} \mathbf{F}_{m}^{H}\right) \mathbf{n}_{k+1}}_{\text {inter-user interference }}+\underbrace{\left(\mathbf{u}_{i}^{(j)}\right)^{H} \mathbf{n}_{1}}_{\text {noise at the destination }} .
\end{aligned}
$$

\section{Channel Model}

Unlike [5], [6], and [21], where the exact CSI is perfectly known, in this paper, we investigate the MAC-BC duality under imperfect CSI at each hop. There are two classes of models frequently used to model imperfect CSI: the Bayesian (stochastic) and the deterministic (or worst-case) models. In the stochastic model, the channel is usually modeled as a complex random matrix with normally distributed elements. The system design is then based on optimizing the stochastic measure of the system performance, such as the mean or 
outage performance under the assumption that the transmitter knows the mean and/or the covariance. On the other hand, the worst-case model assumes that the instantaneous channel, though not exactly known, lies in a known set of possible values. The error belongs to a predefined uncertainty region (with no inherent statistical assumption). In this case, the final objective is to optimize the worst system performance in this error region, which leads to a maximin formulation. In this paper, we consider the stochastic model, where the true CSI at each hop is modeled as

$$
\begin{array}{rlrl}
\mathbf{H}_{l} & =\hat{\mathbf{R}}_{l}^{\frac{1}{2}} \mathbf{H}_{w, l} \mathbf{T}_{l}^{\frac{1}{2}}, \quad l=1, \ldots, L-1 \\
\mathbf{H}_{L_{i}} & =\hat{\mathbf{R}}_{L_{i}}^{\frac{1}{2}} \mathbf{H}_{w, L_{i}} \mathbf{T}_{L_{i}}^{\frac{1}{2}}, & i=1, \ldots, K
\end{array}
$$

where the elements of $\mathbf{H}_{w, l}$ and $\mathbf{H}_{w, L_{i}}$ are i.i.d. zero mean circularly symmetric complex Gaussian random variables all with unit variance, $\mathbf{T}_{l} \in \mathcal{C}^{N_{l} \times N_{l}}$ and $\hat{\mathbf{R}}_{l} \in \mathcal{C}^{N_{l+1} \times N_{l+1}}$ are (from the perspective of BC system) antenna correlation matrices at the transmitter end of the $l$ th node and the receiver end of the $(l+1)$-th node, respectively [26], [27]. Similarly, $\mathbf{T}_{L_{i}} \in \mathcal{C}^{N_{L} \times N_{L}}$ and $\hat{\mathbf{R}}_{L_{i}} \in \mathcal{C}^{N_{L+1}^{(i)} \times N_{L+1}^{(i)}}$ are antenna correlation matrices at the transmitter end of the $(L-1)$-th relay node and the $i$ th user node, respectively.

We assume that channel estimation is performed on $\mathbf{H}_{w, l}$ and $\mathbf{H}_{w, L_{i}}$ using the orthogonal training method developed in [26]. Based on (12) and (13), the true channels $\mathbf{H}_{l}, \mathbf{H}_{L_{i}}$, and their minimum mean-squared error (MMSE) estimates $\tilde{\mathbf{H}}_{l}, \tilde{\mathbf{H}}_{L_{i}}$ are related as [26]

$$
\begin{aligned}
& \mathbf{H}_{l}=\tilde{\mathbf{H}}_{l}+\mathbf{R}_{l}^{\frac{1}{2}} \mathbf{E}_{w, l} \mathbf{T}_{l}^{\frac{1}{2}}=\tilde{\mathbf{H}}_{l}+\mathbf{E}_{l}, \quad l=1, \ldots, L-1 \\
& \mathbf{H}_{L_{i}}=\tilde{\mathbf{H}}_{L_{i}}+\mathbf{R}_{L_{i}}^{\frac{1}{2}} \mathbf{E}_{w, L_{i}} \mathbf{T}_{L_{i}}^{\frac{1}{2}}=\tilde{\mathbf{H}}_{L_{i}}+\mathbf{E}_{L_{i}}, i=1, \ldots, K
\end{aligned}
$$

where $\mathbf{R}_{l} \triangleq\left(\mathbf{I}_{N_{l+1}}+\sigma_{e, l}^{2} \hat{\mathbf{R}}_{l}^{-1}\right)^{-1}, \mathbf{E}_{l} \triangleq \mathbf{R}_{l}^{\frac{1}{2}} \mathbf{E}_{w, l} \mathbf{T}_{l}^{\frac{1}{2}}$ is the estimation error of $\mathbf{H}_{l}, \mathbf{R}_{L_{i}} \triangleq\left(\mathbf{I}_{N_{L+1}^{(i)}}+\sigma_{e, L_{i}}^{2} \hat{\mathbf{R}}_{L_{i}}^{-1}\right)^{-1}$ and $\mathbf{E}_{L_{i}} \triangleq \mathbf{R}_{L_{i}}^{\frac{1}{2}} \mathbf{E}_{w, L_{i}} \mathbf{T}_{L_{i}}^{\frac{1}{2}}$ is the estimation error of $\mathbf{H}_{L_{i}}$. Here, the entries of $\mathbf{E}_{w, l}$ and $\mathbf{E}_{w, L_{i}}$ are i.i.d. with $\mathcal{C N}\left(0, \sigma_{e, l}^{2}\right)$ and $\mathcal{C N}\left(0, \sigma_{e, L_{i}}^{2}\right)$, respectively. For the ease of explanation, let us take the BC system as an example. We assume that the $l$ th node $((l-1)$-th relay node $), l=2, \ldots, L$, estimates its backward channel $\mathbf{H}_{l-1}$ through channel training and feeds the estimated CSI back to the $(l-1)$-th node without any error. Thus, both the $l$ th and the $(l-1)$-th nodes have the same CSI mismatch on $\mathbf{H}_{l-1}$. Similarly, the $i$ th user node estimates its backward channel $\mathbf{H}_{L_{i}}$ through channel training and feeds the estimated CSI back to the $(L-1)$-th relay node without any error. In this paper, we assume that $\mathbf{E}_{w, l}$ is unknown, but $\tilde{\mathbf{H}}_{l}, \mathbf{R}_{l}, \mathbf{T}_{l}$, and $\sigma_{e, l}^{2}$ are available at the $l$ th and the $(l+1)$-th nodes, $l=$ $1, \ldots, L-1$. Similarly, we assume that $\mathbf{E}_{w, L_{i}}$ is unknown, but $\tilde{\mathbf{H}}_{L_{i}}, \mathbf{R}_{L_{i}}, \mathbf{T}_{L_{i}}$, and $\sigma_{e, L_{i}}^{2}$ are available at the $(L-1)$-th relay node and $i$ th user node, $i=1, \ldots, K$.

\section{MAC-BC DUALITY}

In this section, we establish the duality between the MAC and BC multihop AF MIMO relay systems under imperfect CSI and antenna correlation at each hop. We define duality as the achievement of identical stream-wise SINR (MSE, MI) at the MAC and the BC systems with the same amount of total network transmission power under imperfect CSI. ${ }^{2}$ In order to establish this duality, given a MAC MIMO relay system, we need to determine the scaling factors $c_{l}, l=1, \ldots, L-1$, of the relay amplifying matrix $\mathbf{F}_{l}$ and the source power allocation matrix $\mathbf{Q}$ in the $\mathrm{BC}$ system.

Note that for the simplicity of presenting the proof of the duality results in this paper, we analyze the duality for the single user case. Let us group all users together in $\mathrm{BC}$ and MAC to form one "super" destination node and one "super" source node with $N_{L+1}$ antennas, respectively. The $\mathrm{BC}$ system and the MAC system can be equivalently viewed as a single-user downlink and uplink multihop MIMO relay system, respectively. The following theorem establishes the MAC-BC duality of multihop MIMO relay communication system under imperfect channel model.

Theorem 1: Let $\mathbf{F}_{l}^{H}$ and $c_{l} \mathbf{F}_{l}$ be the relay amplifying matrices at the multihop MIMO MAC and BC systems, respectively. Under imperfect CSI, stream-wise identical estimated SINRs (duality) in the MAC and BC systems can be achieved through the following two approaches:

1) With transmission power constraint at individual nodes, duality holds by setting $P_{L+1-l}^{M}=P_{l}^{B}, l=1, \ldots, L$, where $P_{l}^{B}$ and $P_{l}^{M}$ are the total transmitted powers at the $l$ th $\mathrm{BC}$ and MAC node, respectively. The values of $c_{l}, l=1, \ldots, L-1$, can be obtained from relay transmission power constraints of the BC. In other words, duality can be achieved by employing $\mathbf{F}_{L-l}^{H}$ and $c_{l} \mathbf{F}_{l}$ respectively as the relay amplifying matrix at the $l$ th relay node of the MAC system and the BC system, $l=1, \ldots, L-1$, and the scaling factor $c_{l}$ is obtained by switching the power constraints at the $l$ th node of the BC system and the $(L+1-l)$-th node of the MAC system, $l=1 \ldots, L$.

2) Under a total network power constraint, MAC-BC duality holds when $\mathbf{F}_{l}$ and $\mathbf{F}_{l}^{H}, l=1, \ldots, L-1$ are the relay amplifying matrices used in the $\mathrm{BC}$ and $\mathrm{MAC}$, respectively. In other words, MAC-BC duality holds when $c_{l}=1, l=1, \ldots, L-1$.

Proof: See Appendix A.

Theorem 1 includes the SINR duality results in [1]-[6], [8] as special cases. It extends the MAC-BC SINR duality results to multihop AF MIMO relay systems under imperfect CSI, and thus generalizes all previous SINR duality results. Note that Theorem 1 holds for any linear transceiver matrices $\mathbf{U}, \mathbf{V}$, and linear relay amplifying matrices $\mathbf{F}_{l}, l=1, \ldots, L-1$.

Theorem 2: If the source node of the BC MIMO relay system employs DPC and the destination node of the MAC MIMO relay system employs SIC, then under imperfect CSI, stream-wise identical estimated SINRs (duality) in the MAC and BC systems can be achieved through the same two approaches in Theorem 1.

Proof: See Appendix B.

\footnotetext{
${ }^{2}$ As described in the Introduction, in this paper we define the SINR as an estimated SINR, which is a ratio of signal power coupled with the estimated channel over interference-plus-noise power plus the channel estimation error variance coupled with the signal power. See (28) and (32) of Appendix A for $\mathrm{BC}$ and MAC, respectively.
} 
Theorem 2 extends the duality results in Theorem 1 to the scenario where nonlinear transceivers are used at the source node of the BC system and the destination node of the MAC system. Similar to Theorem 1, Theorem 2 holds for any transceiver matrices $\mathbf{U}, \mathbf{V}$, and relay amplifying matrices $\mathbf{F}_{l}, l=1, \ldots, L-1$. By choosing $\mathbf{V}$ (the destination receiving matrix in the $\mathrm{BC}$ and the source precoding matrix in the MAC) as a block diagonal matrix, i.e., $\mathbf{V}=\operatorname{bd}\left(\mathbf{V}_{1}, \mathbf{V}_{2}, \ldots, \mathbf{V}_{K}\right)$, both Theorem 1 and Theorem 2 are applicable to multiuser MIMO relay scenario. Moreover, the two duality results are proved under the fair condition that MAC and BC systems consume the same amount of total transmission power.

We can also derive the MSE duality based on the SINR duality by using the relation of $\mathrm{MSE}_{i}^{B}=1 /\left(1+\operatorname{SINR}_{i}^{B}\right)$, $i=1, \ldots, N_{b}$, as shown in Appendix C, where $\mathrm{MSE}_{i}^{B}$ and $\mathrm{SINR}_{i}^{B}$ stand for the MSE and SINR of the $i$ th data stream in the BC system, respectively. Thus identical SINR values in the MAC and BC systems imply identical MSE values. Therefore, it can be concluded from Theorem 1 that each MSE point achievable in the MAC system can be attained in the BC system. Clearly, the converse holds as well.

Based on the SINR duality $\operatorname{SINR}_{i}^{M}=\operatorname{SINR}_{i}^{B}, i=$ $1, \ldots, N_{b}$, where $\operatorname{SINR}_{i}^{M}$ is the SINR of the $i$ th data stream of the MAC system. we can prove the MAC-BC MI (or achievable sum-rate) duality as

$$
\begin{aligned}
C^{M} & =\sum_{i=1}^{N_{b}} \log _{2}\left(1+\operatorname{SINR}_{i}^{M}\right) \\
& =\sum_{i=1}^{N_{b}} \log _{2}\left(1+\operatorname{SINR}_{i}^{B}\right) \\
& =C^{B}
\end{aligned}
$$

where $C^{M}$ and $C^{B}$ are the MI of the MAC and BC systems, respectively.

\section{NumERiCAl Results}

The implication of the two duality results is that the achievable SINR (MSE, MI) regions in both MAC and BC systems are the same, i.e. the set of SINR (MSE, MI) is achievable in the BC if and only if it is achievable in the MAC. As a direct application of these duality results, the complicated BC MIMO multihop relay system optimization problems can be carried out efficiently by focusing on an equivalent MAC MIMO multihop relay system. For example, the optimal source precoding matrix and the optimal relay amplifying matrices for MAC multihop MIMO AF relay system with a linear receiver at the destination node is considered in [28]. The multihop AF-MIMO relay system with a nonlinear receiver (SIC) at the destination node is considered in [29]. Although [28] and [29] focus on MAC (uplink) system, an optimal design of the source precoding matrix and relay amplifying matrices for $\mathrm{BC}$ (downlink) multihop AF-MIMO relay systems can be designed by exploiting the MAC-BC duality results for multihop MIMO AF relay systems established in this paper.

The optimal multihop MIMO relay design problem for BC can be formulated as

$$
\begin{aligned}
\min _{\mathbf{U}, \mathbf{Q},\left\{c_{l} \mathbf{F}_{l}\right\}} & f\left(\mathrm{SINR}_{i}^{B}\right) \\
\text { s.t } & P_{l}^{B} \leq \rho_{l}^{B}, \quad l=1, \ldots, L \\
\text { or } \quad \text { s.t } & P_{T}^{B} \leq \rho^{B}
\end{aligned}
$$

where $f($.$) stands for a unified objective function [30],$ and $\operatorname{SINR}_{i}^{B}$ highlighting the imperfect CSI assumption is a function of $\mathbf{U}, \mathbf{Q},\left\{c_{l} \mathbf{F}_{l}\right\}$ given in (28) of Appendix A. $P_{l}^{B}, l=1, \ldots, L$ and $P_{T}^{B}$ are the power consumed by the $l$ th node and the total power consumed in the BC system given by (24)-(26) and (27) of Appendix A, respectively. Here $\rho_{l}^{B}, l=1, \ldots, L$ and $\rho^{B}$ are the individual power constraint at the $l$ th node and the total power constraint of the BC system, respectively. Function $f($.$) includes a broad class of frequently$ used objective functions in MIMO system design such as the negative source-destination mutual information, and the MSE of the signal waveform estimation at the destination.

We apply the theorems established in this paper to optimize BC MIMO multihop relay system. The optimization problem for the dual MAC MIMO multihop relay system can be written as

$$
\begin{aligned}
\min _{\mathbf{V}, \mathbf{P},\left\{\mathbf{F}_{L-l}^{H}\right\}} & f\left(\mathrm{SINR}_{i}^{M}\right) \\
\text { s.t } & P_{l}^{M} \leq \rho_{l}^{M}, \quad l=1, \ldots, L \\
\text { or } \quad \text { s.t } & \sum_{l=1}^{L} P_{l}^{M} \leq \rho^{M}
\end{aligned}
$$

where $\operatorname{SINR}_{i}^{M}$ highlighting the imperfect CSI assumption is a function of $\mathbf{V}, \mathbf{P},\left\{\mathbf{F}_{L-l}^{H}\right\}$ given in (32) of Appendix A. $P_{l}^{M}, l=1, \ldots, L$ is the power consumed by the $l$ th node in the MAC system given by (21)-(23) of Appendix A. Here $\rho_{l}^{M}, l=1, \ldots, L$ and $\rho^{M}$ are the individual power constraint at the $l$ th node and the total power constraint of the MAC system, respectively.

As an example of the duality results established here, we consider the sum-MSE optimization problem for multihop MIMO relay systems under imperfect CSI through numerical simulations. We simulate a flat Rayleigh fading environment where all channel matrices have entries with zero mean. The variance of entries in $\mathbf{H}_{L_{i}}$ is $1 / N_{L+1}^{(i)}, i=1, \cdots, K$, and the variance of entries in $\mathbf{H}_{l}$ is $1 / N_{l}, l=1, \cdots, L-1$. All noises are complex circularly symmetric with zero mean and unit variance.

All simulation results are averaged over 1000 independent channel realizations. We use the iterative algorithm in [31] to design the optimal MAC parameters $\mathbf{F}_{l}^{H}, l=1, \ldots, L-1$, $\mathbf{V}_{j}, j=1, \ldots, K, \mathbf{P}_{j}, j=1, \ldots, K$ and use the proposed duality theorem to obtain the dual BC parameters $c_{l} \mathbf{F}_{l}, l=$ $1, \ldots, L-1, \mathbf{U}_{j}, j=1, \ldots, K, \mathbf{Q}_{j}, j=1, \ldots, K^{3}$. For all examples, we set $P_{L}^{M}=P_{1}^{B}=20 \mathrm{~dB}$ and assume that $P_{l}^{B}=P_{L-l+1}^{M}=P, l=2, \ldots, L$ for simplicity.

We simulate five-hop multiuser MIMO relay systems in our examples. Since there are many parameters on the system

\footnotetext{
${ }^{3}$ The proposed algorithm in [31] holds for both the cases with and without perfect CSI.
} 


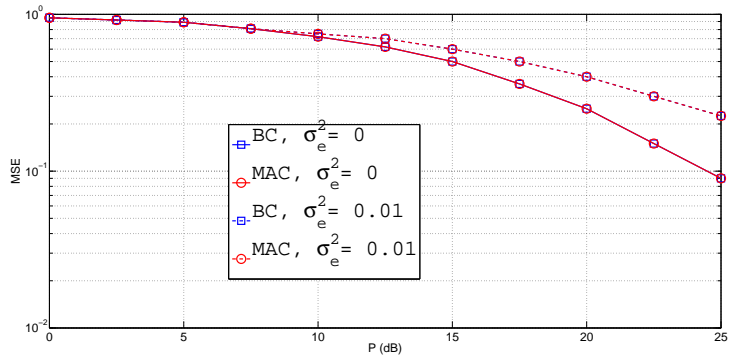

Fig. 3. MSE versus $P$. $K=3, M=2, N=10, P_{L}^{M}=P_{1}^{B}=20 \mathrm{~dB}$.

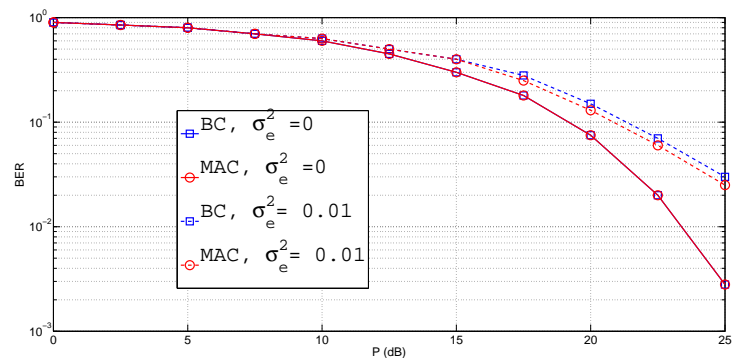

Fig. 4. BER versus $P . K=3, M=2, N=10, P_{L}^{M}=P_{1}^{B}=20 \mathrm{~dB}$.

setup for multihop relays, for simplicity, we consider relay systems where all users have the same number of antennas (i.e., $N_{L+1}^{(i)}=M, i=1, \cdots, K$ ) and all relay nodes and the destination node in the MAC have the same number of antennas (i.e., $N_{l}=N, l=1, \cdots, L$ ). The extension to systems where different nodes have different number of antennas is straightforward. Moreover, we assume that channel estimation errors have the same variance, i.e., $\sigma_{e, l}^{2}=\sigma_{e, L_{i}}^{2}=\sigma_{e}^{2}$, $l=1, \cdots, L-1, i=1, \cdots, K$. Fig. 3 shows the MSE performance of the MAC and BC systems versus $P$ with $K=3, M=2$, and $N=10$. It can be clearly seen from Fig. 3 that the curves overlap, indicating that both the MAC and BC systems achieve the same sum-MSE under both perfect CSI and imperfect CSI. The BER performance of both systems with QPSK constellations is illustrated in Fig. 4 versus $P$. It can be clearly seen from Fig. 4 that under perfect CSI the curves overlap, indicating that both the MAC and BC systems achieve the same BER. However, under imperfect CSI, the curves diverge slightly when $P$ is large. The reason for the divergence is explained in [8] and [17] that the derivations of SINRs or MSEs depend largely on the first-order and secondorder statistics of transmitted signal, fading, channel noise and channel estimation error, which are the same for both BC and MAC systems. Therefore, it is expected that the MSEs of both links seem to be the same. On the other hand, the BERs depend on the interference-plus-noise distributions, which vary with the relative strengths of the interferences. When there is channel estimation error, the interference strengths depend on $P$ and this dependence is possibly different for the MAC and BC. Therefore, the BERs become noticeably unequal when $P$ is relatively large (and thus channel estimation error becomes the dominant source of errors). Similar results can also be obtained for other transceiver design approaches.

\section{CONCLUSION}

We have investigated the MAC-BC SINR (MSE, MI) duality in a multihop AF MIMO relay system under imperfect CSI and antenna correlation at each hop, which is a generalization of several previously established results. We proved that identical stream-wise SINR (MSE, MI) in the MAC and BC systems can be achieved by two approaches. Firstly, under the same total network transmission power constraint, the relay nodes of the BC system employ the Hermitian transposed MAC system relay amplifying matrices. Secondly, under the individual transmission power constraint at each node of the system, the relays of the $\mathrm{BC}$ system use the scaled and Hermitian transposed MAC system relay amplifying matrices, where the scaling factors are obtained by swapping the power constraints of the nodes of the MAC system.

\section{APPENDIX A \\ PROOF OF THEOREM 1}

In order to establish the SINR duality for multihop AF MIMO relay systems under imperfect CSI, we have to show the conditions on $\mathbf{P}, \mathbf{Q}$, and $c_{l}, l=1, \ldots, L-1$, that identical estimated SINRs are achieved in both the MAC and $\mathrm{BC}$ systems. The proof consists of the three main steps.

1) We write the total transmission power and the estimated SINR of each stream for both the MAC and BC systems using (8)-(11) and (3)-(7), respectively.

2) We rewrite the total transmission power of the BC system obtained in Step 1 based on the definition of duality that both channels should achieve identical SINRs.

3) Using the final expression of the total transmission power of the BC system obtained in Step 2, we find the conditions on $\mathbf{P}, \mathbf{Q}$, and $c_{l}, l=1, \ldots, L-1$, such that both the MAC and BC systems consume the same amount of total transmission power.

\section{A. Step 1}

In this step, we first write the total required transmission power for the MAC and BC systems. For this purpose, we first express the transmitted power at each node of both systems.

Using (8) and (9), the individual transmission power for all the transmitting nodes in the MAC system can be written as

$$
\begin{aligned}
& P_{1}^{M}= \operatorname{tr}\{\mathbf{P}\} \\
& P_{2}^{M}=\mathbb{E}[\left.\operatorname{tr}\left\{\mathbf{F}_{L-1}^{H}\left(\mathbf{H}_{L}^{H} \mathbf{V} \mathbf{P} \mathbf{V}^{H} \mathbf{H}_{L}+\mathbf{I}_{N_{L}}\right) \mathbf{F}_{L-1}\right\}\right] \\
&= \operatorname{tr}\left\{\mathbf{F}_{L-1}^{H} \mathbf{F}_{L-1}\right\}+\operatorname{tr}\left\{\left(\tilde{\mathbf{H}}_{L} \mathbf{F}_{L-1} \mathbf{F}_{L-1}^{H} \tilde{\mathbf{H}}_{L}^{H}\right.\right. \\
&\left.\left.+\sigma_{e, L}^{2} \operatorname{tr}\left\{\mathbf{T}_{L} \mathbf{F}_{L-1} \mathbf{F}_{L-1}^{H}\right\} \mathbf{R}_{L}\right) \mathbf{V P} \mathbf{V}^{H}\right\} \\
& P_{L+2-l}^{M}=\mathbb{E}[\left.\operatorname{tr}\left\{\mathbf{F}_{l-1}^{H} \mathbf{y}_{L+2-l}^{M}\left(\mathbf{y}_{L+2-l}^{M}\right)^{H} \mathbf{F}_{l-1}\right\}\right] \\
&= \operatorname{tr}\left\{\left(\tilde{\mathbf{H}}_{L} \mathbf{F}_{L-1} \mathbf{B}_{L}^{(l)} \mathbf{F}_{L-1}^{H} \tilde{\mathbf{H}}_{L}^{H}\right.\right. \\
&+\sigma_{e, L}^{2} \operatorname{tr}\left\{\mathbf{T}_{L} \mathbf{F}_{L-1} \mathbf{B}_{L}^{(l)} \mathbf{F}_{L-1}^{H}\right\} \\
&\left.\left.\mathbf{R}_{L}\right) \mathbf{V P V} \mathbf{V}^{H}\right\} \\
& \underbrace{\sum_{k=l} \operatorname{tr}\left\{\mathbf{F}_{l-1}^{H} \mathbf{D}_{l-1}^{(k)} \mathbf{F}_{l-1}\right\}}_{\text {propagated noise power }}+\underbrace{t r\left\{\mathbf{F}_{l-1}^{H} \mathbf{F}_{l-1}\right\}}_{\text {noise power }}, l=2, \ldots, L-1
\end{aligned}
$$


where $\mathbf{B}_{m}^{(n)}$ and $\mathbf{D}_{m}^{(n)}$ are recursive functions and given by

$\mathbf{B}_{m}^{(n)}=\left\{\begin{array}{lc}\mathbf{I}_{N_{n}}, & \text { if } m=n \\ \tilde{\mathbf{H}}_{m-1} \mathbf{F}_{m-2} \mathbf{B}_{m-1}^{(n)} \mathbf{F}_{m-2}^{H} \tilde{\mathbf{H}}_{m-1}^{H} & \\ +\sigma_{e, m-1}^{2} \operatorname{tr}\left\{\mathbf{T}_{m-1} \mathbf{F}_{m-2} \mathbf{B}_{m-1}^{(n)} \mathbf{F}_{m-2}^{H}\right\} \mathbf{R}_{m-1}, & \text { o.w. }\end{array}\right.$

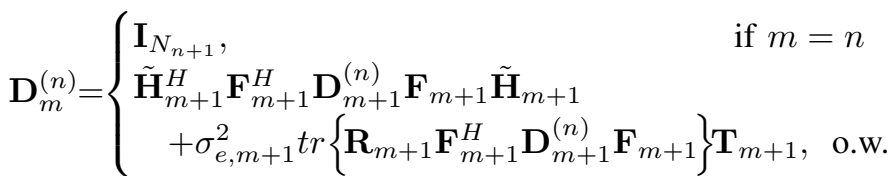

The total transmission power of the MAC system $P_{T}^{M}$ can be calculated with the summation of all individual powers (21)(23). We would like to note that since the exact CSI is unknown, the transmission power is averaged over the imperfect CSI through the expectation operations in (22) and (23) with respect to $\mathbf{E}_{w, l}$. Here, we have used the result of $\mathbb{E}\left[\mathbf{E} \mathbf{A} \mathbf{E}^{H}\right]=\sigma_{e}^{2} \operatorname{tr}\{\mathbf{A}\} \mathbf{I}$, when the entries of $\mathbf{E}$ are i.i.d. with $\mathcal{C N}\left(0, \sigma_{e}^{2}\right)$ and $\mathbf{A}$ is a given matrix [26].

Using (3) and (4), the individual transmission power $P_{l}^{B}$ of the $l$ th node, $l=1, \ldots, L$, in the $\mathrm{BC}$ system can be written as

$$
\begin{aligned}
& P_{1}^{B}= \operatorname{tr}\{\mathbf{Q}\} \\
& P_{2}^{B}=\mathbb{E}\left.\operatorname{tr}\left\{c_{1}^{2} \mathbf{F}_{1}\left(\mathbf{H}_{1} \mathbf{U} \mathbf{Q} \mathbf{U}^{H} \mathbf{H}_{1}^{H}+\mathbf{I}_{N_{2}}\right) \mathbf{F}_{1}^{H}\right\}\right] \\
&= \operatorname{tr}\left\{c_{1}^{2} \mathbf{F}_{1} \mathbf{F}_{1}^{H}\right\}+c_{1}^{2} \operatorname{tr}\left\{\left(\tilde{\mathbf{H}}_{1}^{H} \mathbf{F}_{1}^{H} \mathbf{F}_{1} \tilde{\mathbf{H}}_{1}\right.\right. \\
&\left.\left.+\sigma_{e, 1}^{2} \operatorname{tr}\left\{\mathbf{R}_{1} \mathbf{F}_{1}^{H} \mathbf{F}_{1}\right\} \mathbf{T}_{1}\right) \mathbf{U} \mathbf{Q} \mathbf{U}^{H}\right\} \\
& P_{l+1}^{B}=\mathbb{E}\left[c_{l}^{2} \operatorname{tr}\left\{\mathbf{F}_{l} \mathbf{y}_{l+1}^{B}\left(\mathbf{y}_{l+1}^{B}\right)^{H} \mathbf{F}_{l}^{H}\right\}\right] \\
&=\left(\prod_{m=1}^{l} c_{m}^{2}\right) \operatorname{tr}\left\{\left(\tilde{\mathbf{H}}_{1}^{H} \mathbf{F}_{1}^{H} \mathbf{D}_{1}^{(l)} \mathbf{F}_{1} \tilde{\mathbf{H}}_{1}\right.\right. \\
&\left.\left.+\sigma_{e, 1}^{2} \operatorname{tr}\left\{\mathbf{R}_{1} \mathbf{F}_{1}^{H} \mathbf{D}_{1}^{(l)} \mathbf{F}_{1}\right\} \mathbf{T}_{1}\right) \mathbf{U Q U}^{H}\right\} \\
&+\underbrace{\sum_{k=2}^{l}\left(\prod_{m=k-1}^{l} c_{m}^{2}\right) \operatorname{tr}\left\{\mathbf{F}_{l} \mathbf{B}_{l+1}^{(k)} \mathbf{F}_{l}^{H}\right\}}_{\text {propagated noise power }} \\
&+\underbrace{\operatorname{tr}\left\{c_{l}^{2} \mathbf{F}_{l} \mathbf{F}_{l}^{H}\right\}}_{\text {noise power }}, l=2, \ldots, L-1 .
\end{aligned}
$$

Note that the expectations in (25) and (26) are taken with respect to $\mathbf{E}_{w, l}$. The total transmission power of the $\mathrm{BC}$ system can be calculated using (24)-(26) and given by

$$
\begin{aligned}
& P_{T}^{B} \\
& =P_{1}^{B}+P_{2}^{B}+\sum_{l=2}^{L-1} P_{l+1}^{B} \\
& =\operatorname{tr}\left\{\mathbf{U} \mathbf{Q} \mathbf{U}^{H}\right\}+\sum_{l=1}^{L-1}\left(\prod_{m=1}^{l} c_{m}^{2}\right) \operatorname{tr}\left\{\left(\tilde{\mathbf{H}}_{1}^{H} \mathbf{F}_{1}^{H} \mathbf{D}_{1}^{(l)} \mathbf{F}_{1} \tilde{\mathbf{H}}_{1}\right.\right. \\
& \left.\left.+\sigma_{e, 1}^{2} \operatorname{tr}\left\{\mathbf{R}_{1} \mathbf{F}_{1}^{H} \mathbf{D}_{1}^{(l)} \mathbf{F}_{1}\right\} \mathbf{T}_{1}\right) \mathbf{U} \mathbf{Q} \mathbf{U}^{H}\right\}+\sum_{l=1}^{L-1} \operatorname{tr}\left\{c_{l}^{2} \mathbf{F}_{l} \mathbf{F}_{l}^{H}\right\} \\
& +\sum_{l=2}^{L-1} \sum_{k=2}^{l}\left(\prod_{m=k-1}^{l} c_{m}^{2}\right) \operatorname{tr}\left\{\mathbf{F}_{l} \mathbf{B}_{l+1}^{(k)} \mathbf{F}_{l}^{H}\right\} .
\end{aligned}
$$

Then we write the estimated SINR of each stream for the MAC and BC systems. Estimated SINR is defined as the ratio of the signal power to the summation of the interference power (interference from all other data streams), total noise power (propagated noise from previous hops plus the thermal noise at the destination node), and the residual power of the signal due to the channel estimation error. By using (7), the estimated SINR of the $i$ th data stream, $i=1, \ldots, N_{b}$ at the destination node of the BC system is given by ${ }^{4}$

$$
\operatorname{SINR}_{i}^{B}=\frac{q_{i}\left(\prod_{m=1}^{L-1} c_{m}^{2}\right)\left|\mathbf{v}_{i}^{H} \tilde{\mathbf{H}}_{L} \bigotimes_{l=L-1}^{1}\left(\mathbf{F}_{l} \tilde{\mathbf{H}}_{l}\right) \mathbf{u}_{i}\right|^{2}}{P_{I_{i}}^{B}}
$$

where $P_{I_{i}}^{B}$ is the total interference plus noise power of the $i$ th stream, $i=1, \ldots, N_{b}$, in the $\mathrm{BC}$ system, and is shown at the bottom of the following page.

The first term $R_{I_{i}}^{B}$ in $P_{I_{i}}^{B}$ is the residual interference power of the desired signal in (7) stemming from the mismatch between the true and estimated CSI, and is given by

$$
\begin{aligned}
R_{I_{i}}^{B} & q_{i} \prod_{m=1}^{L-1} c_{m}^{2} \mathbf{v}_{i}^{H}\left[\sigma_{e, L}^{2} \operatorname{tr}\left\{\mathbf{T}_{L} \mathbf{F}_{L-1} \mathbf{A}_{L-1}^{(i)} \mathbf{F}_{L-1}^{H}\right\} \mathbf{R}_{L}\right. \\
+ & \tilde{\mathbf{H}}_{L}\left(\sum_{k=1}^{L-2} \sigma_{e, k}^{2} \operatorname{tr}\left\{\mathbf{T}_{k} \mathbf{F}_{k-1} \mathbf{A}_{k-1}^{(i)} \mathbf{F}_{k-1}^{H}\right\}\right. \\
& \bigotimes_{m=L-1}^{k+1}\left(\mathbf{F}_{m} \tilde{\mathbf{H}}_{m}\right) \mathbf{F}_{k} \mathbf{R}_{k} \mathbf{F}_{k}^{H} \bigotimes_{m=k+1}^{L-1}\left(\tilde{\mathbf{H}}_{m}^{H} \mathbf{F}_{m}^{H}\right) \\
& \left.\left.+\sigma_{e, L-1}^{2} \operatorname{tr}\left\{\mathbf{T}_{L-1} \mathbf{F}_{L-2} \mathbf{A}_{L-2}^{(i)} \mathbf{F}_{L-2}^{H}\right\} \mathbf{F}_{L-1} \mathbf{R}_{L-1} \mathbf{F}_{L-1}^{H}\right) \tilde{\mathbf{H}}_{L}^{H}\right] \mathbf{v}_{i}
\end{aligned}
$$

where $\mathbf{A}_{k}^{(j)}$ are recursively defined as

$$
\mathbf{A}_{k}^{(j)}= \begin{cases}\mathbf{F}_{0}^{-1} \mathbf{u}_{j} \mathbf{u}_{j}^{H} \mathbf{F}_{0}^{-H}, & \text { if } k=0 \\ \tilde{\mathbf{H}}_{1} \mathbf{u}_{j} \mathbf{u}_{j}^{H} \tilde{\mathbf{H}}_{1}^{H}+\sigma_{e, 1}^{2} \operatorname{tr}\left\{\mathbf{T}_{1} \mathbf{u}_{j} \mathbf{u}_{j}^{H}\right\} \mathbf{R}_{1}, & \text { if } k=1 \\ \tilde{\mathbf{H}}_{k} \mathbf{F}_{k-1} \mathbf{A}_{k-1}^{(j)} \mathbf{F}_{k-1}^{H} \tilde{\mathbf{H}}_{k}^{H} & \\ \quad+\sigma_{e, k}^{2} \operatorname{tr}\left\{\mathbf{T}_{k} \mathbf{F}_{k-1} \mathbf{A}_{k-1}^{(j)} \mathbf{F}_{k-1}^{H}\right\} \mathbf{R}_{k}, & \text { if } k \geq 2 .\end{cases}
$$

Here we introduced the matrix $\mathbf{F}_{0}$ in (31) for the simplicity of presentation. In particular, $\mathbf{F}_{0}$ is an invertible matrix that is canceled by $\mathbf{F}_{0}^{-1}$ in $\mathbf{A}_{0}^{(i)}$ when $k=1$ in the second term of (30).

By using (11), the estimated SINR of the $i$ th data stream, $i=1, \ldots, N_{b}$ at the destination node of the MAC MIMO relay channel is given by

$$
\operatorname{SINR}_{i}^{M}=\frac{p_{i}\left|\mathbf{u}_{i}^{H} \bigotimes_{l=1}^{L-1}\left(\tilde{\mathbf{H}}_{l}^{H} \mathbf{F}_{l}^{H}\right) \tilde{\mathbf{H}}_{L}^{H} \mathbf{v}_{i}\right|^{2}}{P_{I_{i}}^{M}}
$$

where $P_{I_{i}}^{M}$ is the total interference plus noise power of the $i$ th stream, $i=1, \ldots, N_{b}$, in the MAC system, and is shown at the bottom of the next page. The first term $R_{I_{i}}^{M}$ in $P_{I_{i}}^{M}$ is

${ }^{4}$ For the stochastic channel estimation error model adopted in Section III, the channel estimation error is seen as noise [8], [26], [32]. 
the residual interference power of the desired signal in (11) stemming from the channel estimation error and is given by

$$
\begin{aligned}
R_{I_{i}}^{M}= & p_{i} \mathbf{u}_{i}^{H}\left[\sigma_{e, 1}^{2} \operatorname{tr}\left\{\mathbf{R}_{1} \mathbf{F}_{1}^{H} \mathbf{C}_{L-2}^{(i)} \mathbf{F}_{1}\right\} \mathbf{T}_{1}\right. \\
& +\sum_{l=2}^{L} \sigma_{e, l}^{2} \operatorname{tr}\left\{\mathbf{R}_{l} \mathbf{F}_{l}^{H} \mathbf{C}_{L-l-1}^{(i)} \mathbf{F}_{l}\right\} \\
& \left.\bigotimes_{k=1}^{l-1}\left(\tilde{\mathbf{H}}_{k}^{H} \mathbf{F}_{k}^{H}\right) \mathbf{T}_{l} \bigotimes_{k=l-1}^{1}\left(\mathbf{F}_{k} \tilde{\mathbf{H}}_{k}\right)\right] \mathbf{u}_{i}
\end{aligned}
$$

where $\mathbf{C}_{k}^{(j)}$ are given by

$$
\mathbf{C}_{k}^{(j)}= \begin{cases}\mathbf{F}_{L}^{-H} \mathbf{v}_{j} \mathbf{v}_{j}^{H} \mathbf{F}_{L}^{-1}, & \text { if } k=-1 \\ \tilde{\mathbf{H}}_{L}^{H} \mathbf{v}_{j} \mathbf{v}_{j}^{H} \tilde{\mathbf{H}}_{L}+\sigma_{e, L}^{2} \operatorname{tr}\left\{\mathbf{R}_{L} \mathbf{v}_{j} \mathbf{v}_{j}^{H}\right\} \mathbf{T}_{L}, & \text { if } k=0 \\ \tilde{\mathbf{H}}_{L-k}^{H} \mathbf{F}_{L-k}^{H} \mathbf{C}_{k-1}^{(j)} \mathbf{F}_{L-k} \tilde{\mathbf{H}}_{L-k} & \\ +\sigma_{e, L-k}^{2} \operatorname{tr}\left\{\mathbf{R}_{L-k} \mathbf{F}_{L-k}^{H} \mathbf{C}_{k-1}^{(j)} \mathbf{F}_{L-k}\right\} \mathbf{T}_{L-k}, \text { if } k \geq 1 .\end{cases}
$$

Similar to $\mathbf{F}_{0}$ in (31), here we introduced the matrix $\mathbf{F}_{L}$ for the simplicity of presentation. In particular, $\mathbf{F}_{L}$ is an invertible matrix that is canceled by $\mathbf{F}_{L}^{-1}$ in $\mathbf{C}_{-1}^{(i)}$ when $l=L$ in the second term of (34).

\section{B. Step 2}

In this step, we rewrite the total transmission power of the BC system obtained in Step 1 based on the definition of the SINR duality.

In order to achieve identical estimated SINRs at the MAC and $\mathrm{BC}$ systems, $\mathrm{SINR}_{i}^{B}=\mathrm{SINR}_{i}^{M}, i=1, \ldots, N_{b}$ must be satisfied. Using (28) and (32), such SINR equality leads to $\prod_{m=1}^{L-1} c_{m}^{2} q_{i} P_{I_{i}}^{M}=p_{i} P_{I_{i}}^{B}$. Summing this over all $N_{b}$ streams, i.e., $\sum_{i=1}^{N_{b}} \operatorname{SINR}_{i}^{B}=\sum_{i=1}^{N_{b}} \operatorname{SINR}_{i}^{M}$, we have

$$
\sum_{i=1}^{N_{b}}\left(\prod_{m=1}^{L-1} c_{m}^{2}\right) q_{i} P_{I_{i}}^{M}=\sum_{i=1}^{N_{b}} p_{i} P_{I_{i}}^{B}
$$

By substituting (29) and (33) into (35), it can be seen that only the last terms (noise terms) in (29) and (33) remain and the other terms related to interference are canceled out, since ${ }^{5}$

$$
\begin{aligned}
& \sum_{i=1}^{N_{b}} \prod_{m=1}^{L-1} c_{m}^{2} q_{i}\left(R_{I_{i}}^{M}+\sum_{j=1, j \neq i}^{N_{b}} p_{j} \mathbf{u}_{i}^{H}\left[\tilde{\mathbf{H}}_{1}^{H} \mathbf{F}_{1}^{H} \mathbf{C}_{L-2}^{(j)} \mathbf{F}_{1} \tilde{\mathbf{H}}_{1}\right.\right. \\
&\left.\left.+\sigma_{e, 1}^{2} \operatorname{tr}\left\{\mathbf{R}_{1} \mathbf{F}_{1}^{H} \mathbf{C}_{L-2}^{(j)} \mathbf{F}_{1}\right\} \mathbf{T}_{1}\right] \mathbf{u}_{i}\right) \\
&=\sum_{i=1}^{N_{b}} p_{i}\left(R_{I_{i}}^{B}+\sum_{j=1, j \neq i}^{N_{b}} q_{j}\left(\prod_{m=1}^{L-1} c_{m}^{2}\right) \mathbf{v}_{i}^{H}\right. \\
& \quad \times\left[\tilde{\mathbf{H}}_{L} \mathbf{F}_{L-1} \mathbf{A}_{L-1}^{(j)} \mathbf{F}_{L-1}^{H} \tilde{\mathbf{H}}_{L}^{H}\right. \\
&\left.\left.+\sigma_{e, L}^{2} \operatorname{tr}\left\{\mathbf{T}_{L} \mathbf{F}_{L-1} \mathbf{A}_{L-1}^{(j)} \mathbf{F}_{L-1}^{H}\right\} \mathbf{R}_{L}\right] \mathbf{v}_{i}\right) .
\end{aligned}
$$

In other words, (35) can be written as

$$
\begin{aligned}
& \sum_{i=1}^{N_{b}} \prod_{m=1}^{L-1} c_{m}^{2} q_{i}\left(\mathbf { u } _ { i } ^ { H } \left[\sum _ { l = 1 } ^ { L - 1 } \left(\tilde{\mathbf{H}}_{1}^{H} \mathbf{F}_{1}^{H} \mathbf{D}_{1}^{(l)} \mathbf{F}_{1} \tilde{\mathbf{H}}_{1}\right.\right.\right. \\
& \left.\left.\left.\quad+\sigma_{e, 1}^{2} \operatorname{tr}\left\{\mathbf{R}_{1} \mathbf{F}_{1}^{H} \mathbf{D}_{1}^{(l)} \mathbf{F}_{1}\right\} \mathbf{T}_{1}\right)\right] \mathbf{u}_{i}+1\right) \\
& =\sum_{i=1}^{N_{b}} p_{i}\left(\mathbf { v } _ { i } ^ { H } \left[\sum _ { l = 2 } ^ { L } \prod _ { m = l - 1 } ^ { L - 1 } c _ { m } ^ { 2 } \left(\tilde{\mathbf{H}}_{L} \mathbf{F}_{L-1} \mathbf{B}_{L}^{(l)} \mathbf{F}_{L-1}^{H} \tilde{\mathbf{H}}_{L}^{H}\right.\right.\right. \\
& \left.\left.\left.\quad+\sigma_{e, L}^{2} \operatorname{tr}\left\{\mathbf{T}_{L} \mathbf{F}_{L-1} \mathbf{B}_{L}^{(l)} \mathbf{F}_{L-1}^{H}\right\} \mathbf{R}_{L}\right)\right] \mathbf{v}_{i}+1\right) .
\end{aligned}
$$

${ }^{5}$ The relation in (36) was used for single-hop, two-hop and multihop channels in [33], [5] and [6], respectively.

$$
\begin{aligned}
P_{I_{i}}^{B}= & R_{I_{i}}^{B}+\underbrace{\sum_{j=1, j \neq i}^{N_{b}} q_{j}\left(\prod_{m=1}^{L-1} c_{m}^{2}\right) \mathbf{v}_{i}^{H}\left[\tilde{\mathbf{H}}_{L} \mathbf{F}_{L-1} \mathbf{A}_{L-1}^{(j)} \mathbf{F}_{L-1}^{H} \tilde{\mathbf{H}}_{L}^{H}+\sigma_{e, L}^{2} \operatorname{tr}\left\{\mathbf{T}_{L} \mathbf{F}_{L-1} \mathbf{A}_{L-1}^{(j)} \mathbf{F}_{L-1}^{H}\right\} \mathbf{R}_{L}\right] \mathbf{v}_{i}}_{\text {interference power }} \\
& +\underbrace{\mathbf{v}_{i}^{H}\left[\sum_{l=2}^{L}\left(\prod_{m=l-1}^{L-1} c_{m}^{2}\right)\left(\tilde{\mathbf{H}}_{L} \mathbf{F}_{L-1} \mathbf{B}_{L}^{(l)} \mathbf{F}_{L-1}^{H} \tilde{\mathbf{H}}_{L}^{H}+\sigma_{e, L}^{2} \operatorname{tr}\left\{\mathbf{T}_{L} \mathbf{F}_{L-1} \mathbf{B}_{L}^{(l)} \mathbf{F}_{L-1}^{H}\right\} \mathbf{R}_{L}\right)+\mathbf{I}_{N_{L}}\right] \mathbf{v}_{i}} .
\end{aligned}
$$

$$
\begin{aligned}
P_{I_{i}}^{M}= & R_{I_{i}}^{M}+\underbrace{\mathbf{u}_{i}^{H} \sum_{j=1, j \neq i}^{N_{b}} p_{j}\left[\tilde{\mathbf{H}}_{1}^{H} \mathbf{F}_{1}^{H} \mathbf{C}_{L-2}^{(j)} \mathbf{F}_{1} \tilde{\mathbf{H}}_{1}+\sigma_{e, 1}^{2} \operatorname{tr}\left\{\mathbf{R}_{1} \mathbf{F}_{1}^{H} \mathbf{C}_{L-2}^{(j)} \mathbf{F}_{1}\right\} \mathbf{T}_{1}\right] \mathbf{u}_{i}}_{\text {interference power }} \\
& +\underbrace{\mathbf{u}_{i}^{H}\left[\sum_{l=1}^{L-1}\left(\tilde{\mathbf{H}}_{1}^{H} \mathbf{F}_{1}^{H} \mathbf{D}_{1}^{(l)} \mathbf{F}_{1} \tilde{\mathbf{H}}_{1}+\sigma_{e, 1}^{2} \operatorname{tr}\left\{\mathbf{R}_{1} \mathbf{F}_{1}^{H} \mathbf{D}_{1}^{(l)} \mathbf{F}_{1}\right\} \mathbf{T}_{1}\right)+\mathbf{I}_{N_{1}}\right] \mathbf{u}_{i}}_{\text {propagated noise power + noise power at the destination }} \\
\triangleq & \mathbf{u}_{i}^{H} \mathbf{M}_{i} \mathbf{u}_{i} .
\end{aligned}
$$


Substituting (37) back into (27), $P_{T}^{B}$ can be written as

$$
\begin{aligned}
P_{T}^{B} & \operatorname{tr}\left\{\mathbf{U Q \mathbf { U } ^ { H } \}}+\sum_{l=1}^{L-1}\left(\prod_{m=1}^{l} c_{m}^{2}\right) \operatorname{tr}\left\{\left(\tilde{\mathbf{H}}_{1}^{H} \mathbf{F}_{1}^{H} \mathbf{D}_{1}^{(l)} \mathbf{F}_{1} \tilde{\mathbf{H}}_{1}\right.\right.\right. \\
& \left.\left.+\sigma_{e, 1}^{2} \operatorname{tr}\left\{\mathbf{R}_{1} \mathbf{F}_{1}^{H} \mathbf{D}_{1}^{(l)} \mathbf{F}_{1}\right\} \mathbf{T}_{1}\right) \mathbf{U} \mathbf{Q} \mathbf{U}^{H}\right\} \\
+ & \sum_{l=2}^{L-1} \sum_{k=2}^{l}\left(\prod_{m=k-1}^{l} c_{m}^{2}\right) \operatorname{tr}\left\{\mathbf{F}_{l} \mathbf{B}_{l+1}^{(k)} \mathbf{F}_{l}^{H}\right\}+\sum_{l=1}^{L-1} \operatorname{tr}\left\{c_{l}^{2} \mathbf{F}_{l} \mathbf{F}_{l}^{H}\right\} \\
- & \left(\prod_{m=1}^{L-1} c_{m}^{2}\right) \operatorname{tr}\left\{\mathbf{U Q} \mathbf{U}^{H}\right\}-\sum_{l=1}^{L-1}\left(\prod_{m=1}^{L-1} c_{m}^{2}\right) \\
& \times \operatorname{tr}\left\{\left(\tilde{\mathbf{H}}_{1}^{H} \mathbf{F}_{1}^{H} \mathbf{D}_{1}^{(l)} \mathbf{F}_{1} \tilde{\mathbf{H}}_{1}\right.\right. \\
& \left.\left.+\sigma_{e, 1}^{2} \operatorname{tr}\left\{\mathbf{R}_{1} \mathbf{F}_{1}^{H} \mathbf{D}_{1}^{(l)} \mathbf{F}_{1}\right\} \mathbf{T}_{1}\right) \mathbf{U} \mathbf{Q} \mathbf{U}^{H}\right\} \\
+ & \sum_{l=2}^{L}\left(\prod_{m=l-1}^{L-1} c_{m}^{2}\right) \operatorname{tr}\left\{\left(\tilde{\mathbf{H}}_{L} \mathbf{F}_{L-1} \mathbf{B}_{L}^{(l)} \mathbf{F}_{L-1}^{H} \tilde{\mathbf{H}}_{L}^{H}\right.\right. \\
& \left.\left.+\sigma_{e, L}^{2} \operatorname{tr}\left\{\mathbf{T}_{L} \mathbf{F}_{L-1} \mathbf{B}_{L}^{(l)} \mathbf{F}_{L-1}^{H}\right\} \mathbf{R}_{L}\right) \mathbf{V P} \mathbf{V}^{H}+\mathbf{P}\right\}
\end{aligned}
$$

where the first four terms are from (27) and the last three terms are from (37).

After some simple manipulations, $P_{T}^{B}$ can be written as

$$
\begin{aligned}
& P_{T}^{B}=\sum_{l=2}^{L}\left(\prod_{m=l-1}^{L-1} c_{m}^{2}\right) \operatorname{tr}\left\{\left(\tilde{\mathbf{H}}_{L} \mathbf{F}_{L-1} \mathbf{B}_{L}^{(l)} \mathbf{F}_{L-1}^{H} \tilde{\mathbf{H}}_{L}^{H}\right.\right. \\
& \left.\left.+\sigma_{e, L}^{2} \operatorname{tr}\left\{\mathbf{T}_{L} \mathbf{F}_{L-1} \mathbf{B}_{L}^{(l)} \mathbf{F}_{L-1}^{H}\right\} \mathbf{R}_{L}\right) \mathbf{V} \mathbf{P} \mathbf{V}^{H}+\mathbf{P}\right\} \\
& +\sum_{l=1}^{L-2}\left(1-\prod_{m=l+1}^{L-1} c_{m}^{2}\right) \operatorname{tr}\left\{( \prod _ { m = 1 } ^ { l } c _ { m } ^ { 2 } ) \left(\tilde{\mathbf{H}}_{1}^{H} \mathbf{F}_{1}^{H} \mathbf{D}_{1}^{(l)} \mathbf{F}_{1} \tilde{\mathbf{H}}_{1}\right.\right. \\
& \left.\left.+\sigma_{e, 1}^{2} \operatorname{tr}\left\{\mathbf{R}_{1} \mathbf{F}_{1}^{H} \mathbf{D}_{1}^{(l)} \mathbf{F}_{1}\right\} \mathbf{T}_{1}\right) \mathbf{U} \mathbf{Q} \mathbf{U}^{H}\right\} \\
& +\left(1-\prod_{m=1}^{L-1} c_{m}^{2}\right) \operatorname{tr}\left\{\mathbf{U Q U}^{H}\right\}+\sum_{l=1}^{L-1} \operatorname{tr}\left\{c_{l}^{2} \mathbf{F}_{l} \mathbf{F}_{l}^{H}\right\} \\
& +\sum_{l=2}^{L-1} \sum_{k=2}^{l}\left(\prod_{m=k-1}^{l} c_{m}^{2}\right) \operatorname{tr}\left\{\mathbf{F}_{l} \mathbf{B}_{l+1}^{(k)} \mathbf{F}_{l}^{H}\right\} .
\end{aligned}
$$

For notational simplicity, for $l=2, \ldots, L-1$, we denote

$$
a_{l} \triangleq \sum_{k=2}^{l}\left(\prod_{m=k-1}^{l} c_{m}^{2}\right) \operatorname{tr}\left\{\mathbf{F}_{l} \mathbf{B}_{l+1}^{(k)} \mathbf{F}_{l}^{H}\right\} .
$$

Using (39) and with some manipulations, we have

$$
\begin{aligned}
& \sum_{l=2}^{L-1} a_{l} \\
& =\sum_{l=2}^{L-1}\left(\prod_{m=l+1}^{L-1} c_{m}^{2}\right) a_{l}+\sum_{l=2}^{L-1}\left(1-\prod_{m=l+1}^{L-1} c_{m}^{2}\right) a_{l} \\
& =\sum_{l=2}^{L-1} \sum_{k=2}^{l}\left(\prod_{m=k-1}^{L-1} c_{m}^{2}\right) \operatorname{tr}\left\{\mathbf{F}_{l} \mathbf{B}_{l+1}^{(k)} \mathbf{F}_{l}^{H}\right\}+\sum_{l=2}^{L-1}\left(1-\prod_{m=l+1}^{L-1} c_{m}^{2}\right) a_{l} \\
& =\sum_{l=2}^{L-1} \sum_{k=l}^{L-1}\left(\prod_{m=l-1}^{L-1} c_{m}^{2}\right) \operatorname{tr}\left\{\mathbf{F}_{k} \mathbf{B}_{k+1}^{(l)} \mathbf{F}_{k}^{H}\right\}+\sum_{l=2}^{L-1}\left(1-\prod_{m=l+1}^{L-1} c_{m}^{2}\right) a_{l}
\end{aligned}
$$

$$
\begin{aligned}
& =\sum_{l=2}^{L-1}\left(\prod_{m=l}^{L} c_{m-1}^{2}\right) \sum_{k=l}^{L-1} \operatorname{tr}\left\{\mathbf{F}_{l-1}^{H} \mathbf{D}_{l-1}^{(k)} \mathbf{F}_{l-1}\right\} \\
& +\sum_{l=2}^{L-1}\left(1-\prod_{m=l+1}^{L-1} c_{m}^{2}\right) a_{l}
\end{aligned}
$$

where we have used the fact that $\operatorname{tr}\left\{\mathbf{F}_{k} \mathbf{B}_{k+1}^{(l)} \mathbf{F}_{k}^{H}\right\}=$ $\operatorname{tr}\left\{\mathbf{F}_{l-1}^{H} \mathbf{D}_{l-1}^{(k)} \mathbf{F}_{l-1}\right\}$. We can also write

$$
\begin{aligned}
& \sum_{l=1}^{L-1} \operatorname{tr}\left\{c_{l}^{2} \mathbf{F}_{l} \mathbf{F}_{l}^{H}\right\} \\
& =\sum_{l=1}^{L-1} \operatorname{tr}\left\{c_{l}^{2} \mathbf{F}_{l} \mathbf{F}_{l}^{H}\right\} \\
& +\underbrace{\sum_{l=2}^{L} \prod_{m=l}^{L} c_{m-1}^{2} \operatorname{tr}\left\{\mathbf{F}_{l-1}^{H} \mathbf{F}_{l-1}\right\}-\sum_{l=1}^{L-1}\left(\prod_{m=l}^{L-1} c_{m}^{2}\right) \operatorname{tr}\left\{\mathbf{F}_{l} \mathbf{F}_{l}^{H}\right\}}_{\text {equal to zero }} \\
& =\sum_{l=2}^{L} \prod_{m=l}^{L} c_{m-1}^{2} \operatorname{tr}\left\{\mathbf{F}_{l-1}^{H} \mathbf{F}_{l-1}\right\} \\
& +\sum_{l=1}^{L-1}\left(1-\prod_{m=l+1}^{L-1} c_{m}^{2}\right) \operatorname{tr}\left\{c_{l}^{2} \mathbf{F}_{l} \mathbf{F}_{l}^{H}\right\} .
\end{aligned}
$$

Substituting (40) and (41) back into (38) and after rearranging terms, $P_{T}^{B}$ is shown at the bottom of the next page.

\section{Step 3}

In this step, using the final expression of the total transmission power of the BC system in (42), we find the conditions on $\mathbf{P}, \mathbf{Q}$, and $c_{l}, l=1, \ldots, L-1$, such that both the MAC and $\mathrm{BC}$ systems consume the same amount of total transmission power.

Using the expressions of $P_{l}^{M}$ in (21)-(23) and $P_{l}^{B}$ in (24)(26), $l=1 \ldots, L,(42)$ can be written as

$$
\begin{aligned}
P_{T}^{B} & \sum_{l=2}^{L-1}\left(\prod_{m=l}^{L} c_{m-1}^{2}\right) P_{L+2-l}^{M}+c_{L-1}^{2} P_{2}^{M}+P_{1}^{M} \\
& +\sum_{l=2}^{L-2}\left(1-\prod_{m=l+1}^{L-1} c_{m}^{2}\right) P_{l+1}^{B}+\sum_{l=1}^{2}\left(1-\prod_{m=l}^{L-1} c_{m}^{2}\right) P_{l}^{B} \\
= & \sum_{l=1}^{L-1}\left(\prod_{m=l}^{L-1} c_{m}^{2}\right) P_{L+1-l}^{M}+P_{1}^{M}+\sum_{l=1}^{L-1}\left(1-\prod_{m=l}^{L-1} c_{m}^{2}\right) P_{l}^{B} .
\end{aligned}
$$

By adding and subtracting $\left(\sum_{l=1}^{L-1} P_{L+1-l}^{M}\right)$ to and from (43), which is the total relay transmission power for the MAC system, we obtain ${ }^{6}$

$$
P_{T}^{B}-P_{T}^{M}=\sum_{l=1}^{L-1}\left(\prod_{m=l}^{L-1} c_{m}^{2}-1\right)\left(P_{L+1-l}^{M}-P_{l}^{B}\right) .
$$

${ }^{6} P_{T}^{M}$ on the left hand side of (44) is obtained by adding $\sum_{l=1}^{L-1} P_{L+1-l}^{M}$ and $P_{1}^{M}$ in (43). 
Since the MAC and BC systems should consume the same amount of total transmission power, we need to find the conditions such that $P_{T}^{B}-P_{T}^{M}=0$. Obviously, for any $L \geq 2$, the condition $P_{T}^{B}-P_{T}^{M}=0$ is true if $\prod_{m=l}^{L-1} c_{m}^{2}=1$ for $l=$ $1, \ldots, L-1$, which is equivalent to $c_{l}=1, l=1, \ldots, L-1$. Thus, the first part of Theorem 1 (without transmission power constraint at each node) is proven. Moreover, the condition $P_{T}^{B}-P_{T}^{M}=0$ also holds if $P_{L+1-l}^{M}=P_{l}^{B}, l=1, \ldots, L-1$. Then we have $P_{1}^{M}=P_{L}^{B}$ due to the fair assumption
$P_{T}^{B}=P_{T}^{M}$. Thus, we have $P_{L+1-l}^{M}=P_{l}^{B}, l=1, \ldots, L$ and the second part of Theorem 1 (with transmission power constraint at individual nodes) is proven.

\section{APPENDIX B}

Proof OF THEOREM 2

When the destination node of a MAC MIMO relay system employs SIC, the source symbols are detected successively with the last symbol detected first and the first symbol detected

$$
\begin{aligned}
& P_{T}^{B}=\underbrace{\sum_{l=2}^{L-1} \prod_{m=l}^{L} c_{m-1}^{2} \operatorname{tr}\left\{\left(\tilde{\mathbf{H}}_{L} \mathbf{F}_{L-1} \mathbf{B}_{L}^{(l)} \mathbf{F}_{L-1}^{H} \tilde{\mathbf{H}}_{L}^{H}+\sigma_{e, L}^{2} \operatorname{tr}\left\{\mathbf{T}_{L} \mathbf{F}_{L-1} \mathbf{B}_{L}^{(l)} \mathbf{F}_{L-1}^{H}\right\} \mathbf{R}_{L}\right) \mathbf{V} \mathbf{P} \mathbf{V}^{H}\right\}} \\
& \text { part of first term in (38) } \\
& +\underbrace{c_{L-1}^{2} \operatorname{tr}\left\{\left(\tilde{\mathbf{H}}_{L} \mathbf{F}_{L-1} \mathbf{F}_{L-1}^{H} \tilde{\mathbf{H}}_{L}^{H}+\sigma_{e, L}^{2} \operatorname{tr}\left\{\mathbf{T}_{L} \mathbf{F}_{L-1} \mathbf{F}_{L-1}^{H}\right\} \mathbf{R}_{L}\right) \mathbf{V P} \mathbf{V}^{H}\right\}+\operatorname{tr}\{\mathbf{P}\}} \\
& \text { part of first term in (38), particularly when } l=L \\
& +\underbrace{\sum_{l=2}^{L-2}\left(1-\prod_{m=l+1}^{L-1} c_{m}^{2}\right) \operatorname{tr}\left\{\prod_{m=1}^{l} c_{m}^{2}\left(\tilde{\mathbf{H}}_{1}^{H} \mathbf{F}_{1}^{H} \mathbf{D}_{1}^{(l)} \mathbf{F}_{1} \tilde{\mathbf{H}}_{1}+\sigma_{e, 1}^{2} \operatorname{tr}\left\{\mathbf{R}_{1} \mathbf{F}_{1}^{H} \mathbf{D}_{1}^{(l)} \mathbf{F}_{1}\right\} \mathbf{T}_{1}\right) \mathbf{U Q U} \mathbf{U}^{H}\right\}}_{\text {part of second term in (38) }} \\
& +\underbrace{\left(1-\prod_{m=2}^{L-1} c_{m}^{2}\right) \operatorname{tr}\left\{c_{1}^{2}\left(\tilde{\mathbf{H}}_{1}^{H} \mathbf{F}_{1}^{H} \mathbf{F}_{1} \tilde{\mathbf{H}}_{1}+\sigma_{e, 1}^{2} \operatorname{tr}\left\{\mathbf{R}_{1} \mathbf{F}_{1}^{H} \mathbf{F}_{1}\right\} \mathbf{T}_{1}\right) \mathbf{U Q \mathbf { Q U }}{ }^{H}\right\}} \\
& +\underbrace{\left(1-\prod_{m=1}^{L-1} c_{m}^{2}\right) \operatorname{tr}\left\{\mathbf{U Q \mathbf { U } ^ { H }}\right\}}_{\text {third term in (38) }}+\underbrace{\sum_{l=2}^{L-1} \prod_{m=l}^{L} c_{m-1}^{2} \operatorname{tr}\left\{\mathbf{F}_{l-1}^{H} \mathbf{F}_{l-1}\right\}+c_{L-1}^{2} \operatorname{tr}\left\{\mathbf{F}_{L-1}^{H} \mathbf{F}_{L-1}\right\}}_{\text {first part of the fourth term in (38) given in (41) }} \\
& +\underbrace{\sum_{l=2}^{L-2}\left(1-\prod_{m=l+1}^{L-1} c_{m}^{2}\right) \operatorname{tr}\left\{c_{l}^{2} \mathbf{F}_{l} \mathbf{F}_{l}^{H}\right\}+\left(1-\prod_{m=2}^{L-1} c_{m}^{2}\right) \operatorname{tr}\left\{c_{1}^{2} \mathbf{F}_{1} \mathbf{F}_{1}^{H}\right\}}_{\text {second part of the fourth term in (38) given in (41) }} \\
& +\underbrace{\sum_{l=2}^{L-1} \prod_{m=l}^{L} c_{m-1}^{2} \operatorname{tr}\left\{\sum_{k=l}^{L-1} \mathbf{F}_{l-1}^{H} \mathbf{D}_{l-1}^{(k)} \mathbf{F}_{l-1}\right\}+\sum_{l=2}^{L-2}\left(1-\prod_{m=l+1}^{L-1} c_{m}^{2}\right) \operatorname{tr}\left\{\sum_{k=2}^{l} \prod_{m=k-1}^{l} c_{m}^{2} \mathbf{F}_{l} \mathbf{B}_{l+1}^{(k)} \mathbf{F}_{l}^{H}\right\}}_{\text {last term in (38) }} \\
& =\sum_{l=2}^{L-1} \prod_{m=l}^{L} c_{m-1}^{2} \operatorname{tr}\left\{\left(\tilde{\mathbf{H}}_{L} \mathbf{F}_{L-1} \mathbf{B}_{L}^{(l)} \mathbf{F}_{L-1}^{H} \tilde{\mathbf{H}}_{L}^{H}+\sigma_{e, L}^{2} \operatorname{tr}\left\{\mathbf{T}_{L} \mathbf{F}_{L-1} \mathbf{B}_{L}^{(l)} \mathbf{F}_{L-1}^{H}\right\} \mathbf{R}_{L}\right) \mathbf{V} \mathbf{P} \mathbf{V}^{H}\right. \\
& \left.+\sum_{k=l}^{L-1} \mathbf{F}_{l-1}^{H} \mathbf{D}_{l-1}^{(k)} \mathbf{F}_{l-1}+\mathbf{F}_{l-1}^{H} \mathbf{F}_{l-1}\right\} \\
& +c_{L-1}^{2} \operatorname{tr}\left\{\left(\tilde{\mathbf{H}}_{L} \mathbf{F}_{L-1} \mathbf{F}_{L-1}^{H} \tilde{\mathbf{H}}_{L}^{H}+\sigma_{e, L}^{2} \operatorname{tr}\left\{\mathbf{T}_{L} \mathbf{F}_{L-1} \mathbf{F}_{L-1}^{H}\right\} \mathbf{R}_{L}\right) \mathbf{V P} \mathbf{V}^{H}+\mathbf{F}_{L-1}^{H} \mathbf{F}_{L-1}\right\}+\operatorname{tr}\{\mathbf{P}\} \\
& +\sum_{l=2}^{L-2}\left(1-\prod_{m=l+1}^{L-1} c_{m}^{2}\right) \operatorname{tr}\left\{\prod_{m=1}^{l} c_{m}^{2}\left(\tilde{\mathbf{H}}_{1}^{H} \mathbf{F}_{1}^{H} \mathbf{D}_{1}^{(l)} \mathbf{F}_{1} \tilde{\mathbf{H}}_{1}+\sigma_{e, 1}^{2} \operatorname{tr}\left\{\mathbf{R}_{1} \mathbf{F}_{1}^{H} \mathbf{D}_{1}^{(l)} \mathbf{F}_{1}\right\} \mathbf{T}_{1}\right) \mathbf{U Q U ^ { H }}\right. \\
& \left.+\sum_{k=2}^{l} \prod_{m=k-1}^{l} c_{m}^{2} \mathbf{F}_{l} \mathbf{B}_{l+1}^{(k)} \mathbf{F}_{l}^{H}+c_{l}^{2} \mathbf{F}_{l} \mathbf{F}_{l}^{H}\right\}+\left(1-\prod_{m=1}^{L-1} c_{m}^{2}\right) \operatorname{tr}\left\{\mathbf{U} \mathbf{Q} \mathbf{U}^{H}\right\} \\
& +\left(1-\prod_{m=2}^{L-1} c_{m}^{2}\right) \operatorname{tr}\left\{c_{1}^{2}\left(\tilde{\mathbf{H}}_{1}^{H} \mathbf{F}_{1}^{H} \mathbf{F}_{1} \tilde{\mathbf{H}}_{1}+\sigma_{e, 1}^{2} \operatorname{tr}\left\{\mathbf{R}_{1} \mathbf{F}_{1}^{H} \mathbf{F}_{1}\right\} \mathbf{T}_{1}\right) \mathbf{U} \mathbf{Q} \mathbf{U}^{H}+c_{1}^{2} \mathbf{F}_{1} \mathbf{F}_{1}^{H}\right\} .
\end{aligned}
$$


last, and thus the interference from the previously detected symbols is subtracted to detect the current symbol. Therefore, the estimated SINR of the $i$ th data stream, $i=1, \ldots, N_{b}$ at the MAC MIMO relay system is written as

$$
\operatorname{SINR}_{i}^{M}=\frac{p_{i}\left|\mathbf{u}_{i}^{H} \bigotimes_{l=1}^{L-1}\left(\tilde{\mathbf{H}}_{l}^{H} \mathbf{F}_{l}^{H}\right) \tilde{\mathbf{H}}_{L}^{H} \mathbf{v}_{i}\right|^{2}}{P_{I_{i}}^{M}}
$$

where $P_{I_{i}}^{M}$ is the total interference plus noise power of the $i$ th stream, $i=1, \ldots, N_{b}$, in the MAC system, and can be expressed as

$$
\begin{aligned}
P_{I_{i}}^{M}= & R_{I_{i}}^{M}+\mathbf{u}_{i}^{H} \sum_{j=1}^{i-1} p_{j}\left[\tilde{\mathbf{H}}_{1}^{H} \mathbf{F}_{1}^{H} \mathbf{C}_{L-2}^{(j)} \mathbf{F}_{1} \tilde{\mathbf{H}}_{1}\right. \\
& \left.+\sigma_{e, 1}^{2} \operatorname{tr}\left\{\mathbf{R}_{1} \mathbf{F}_{1}^{H} \mathbf{C}_{L-2}^{(j)} \mathbf{F}_{1}\right\} \mathbf{T}_{1}\right] \mathbf{u}_{i} \\
& +\mathbf{u}_{i}^{H}\left[\sum _ { l = 1 } ^ { L - 1 } \left(\tilde{\mathbf{H}}_{1}^{H} \mathbf{F}_{1}^{H} \mathbf{D}_{1}^{(l)} \mathbf{F}_{1} \tilde{\mathbf{H}}_{1}\right.\right. \\
& \left.\left.+\sigma_{e, 1}^{2} \operatorname{tr}\left\{\mathbf{R}_{1} \mathbf{F}_{1}^{H} \mathbf{D}_{1}^{(l)} \mathbf{F}_{1}\right\} \mathbf{T}_{1}\right)+\mathbf{I}_{N_{1}}\right] \mathbf{u}_{i}
\end{aligned}
$$

where $R_{I_{i}}^{M}$ is defined in (34).

When the source node of a BC MIMO relay system employs DPC, the source symbols are encoded successively with the first symbol encoded first and the last symbol encoded last, and thus the interference from previously encoded symbols is subtracted to encode the current symbol. Therefore, the estimated SINR of the $i$ th data stream, $i=1, \ldots, N_{b}$ at the $\mathrm{BC}$ MIMO relay system is written as

$$
\operatorname{SINR}_{i}^{B}=\frac{q_{i}\left(\prod_{m=1}^{L-1} c_{m}^{2}\right)\left|\mathbf{v}_{i}^{H} \tilde{\mathbf{H}}_{L} \bigotimes_{l=L-1}^{1}\left(\mathbf{F}_{l} \tilde{\mathbf{H}}_{l}\right) \mathbf{u}_{i}\right|^{2}}{P_{I_{i}}^{B}}
$$

where $P_{I_{i}}^{B}$ is the total interference plus noise power of the $i$ th stream, $i=1, \ldots, N_{b}$, in the BC system, and can be written as (using the definition of $R_{I_{i}}^{B}$ in (30))

$$
\begin{aligned}
P_{I_{i}}^{B}= & \sum_{j=i+1}^{N_{b}} q_{j}\left(\prod_{m=1}^{L-1} c_{m}^{2}\right) \mathbf{v}_{i}^{H}\left[\tilde{\mathbf{H}}_{L} \mathbf{F}_{L-1} \mathbf{A}_{L-1}^{(j)} \mathbf{F}_{L-1}^{H} \tilde{\mathbf{H}}_{L}^{H}\right. \\
& \left.+\sigma_{e, L}^{2} \operatorname{tr}\left\{\mathbf{T}_{L} \mathbf{F}_{L-1} \mathbf{A}_{L-1}^{(j)} \mathbf{F}_{L-1}^{H}\right\} \mathbf{R}_{L}\right] \mathbf{v}_{i}+R_{I_{i}}^{B} \\
& +\mathbf{v}_{i}^{H}\left[\sum _ { l = 2 } ^ { L } ( \prod _ { m = l - 1 } ^ { L - 1 } c _ { m } ^ { 2 } ) \left(\tilde{\mathbf{H}}_{L} \mathbf{F}_{L-1} \mathbf{B}_{L}^{(l)} \mathbf{F}_{L-1}^{H} \tilde{\mathbf{H}}_{L}^{H}\right.\right. \\
& \left.\left.+\sigma_{e, L}^{2} \operatorname{tr}\left\{\mathbf{T}_{L} \mathbf{F}_{L-1} \mathbf{B}_{L}^{(l)} \mathbf{F}_{L-1}^{H}\right\} \mathbf{R}_{L}\right)+\mathbf{I}_{N_{L}}\right] \mathbf{v}_{i} .(48)
\end{aligned}
$$

Using (45) and (47), and the identity below (similar to (36)),

$$
\begin{aligned}
& \sum_{i=1}^{N_{b}} \prod_{m=1}^{L-1} c_{m}^{2} q_{i}\left(R_{I_{i}}^{M}+\sum_{j=1}^{i-1} p_{j} \mathbf{u}_{i}^{H}\left[\tilde{\mathbf{H}}_{1}^{H} \mathbf{F}_{1}^{H} \mathbf{C}_{L-2}^{(j)} \mathbf{F}_{1} \tilde{\mathbf{H}}_{1}\right.\right. \\
& \left.\left.+\sigma_{e, 1}^{2} \operatorname{tr}\left\{\mathbf{R}_{1} \mathbf{F}_{1}^{H} \mathbf{C}_{L-2}^{(j)} \mathbf{F}_{1}\right\} \mathbf{T}_{1}\right] \mathbf{u}_{i}\right) \\
& =\sum_{i=1}^{N_{b}} p_{i}\left(R_{I_{i}}^{B}+\sum_{j=i+1}^{N_{b}} q_{j}\left(\prod_{m=1}^{L-1} c_{m}^{2}\right) \mathbf{v}_{i}^{H}\left[\tilde{\mathbf{H}}_{L} \mathbf{F}_{L-1} \mathbf{A}_{L-1}^{(j)} \mathbf{F}_{L-1}^{H} \tilde{\mathbf{H}}_{L}^{H}\right.\right. \\
& \left.\left.\quad+\sigma_{e, L}^{2} \operatorname{tr}\left\{\mathbf{T}_{L} \mathbf{F}_{L-1} \mathbf{A}_{L-1}^{(j)} \mathbf{F}_{L-1}^{H}\right\} \mathbf{R}_{L}\right] \mathbf{v}_{i}\right)
\end{aligned}
$$

we obtain $P_{T}^{B}$ as in (38) from $\sum_{i=1}^{N_{b}} \operatorname{SINR}_{i}^{B}=\sum_{i=1}^{N_{b}} \operatorname{SINR}_{i}^{M}$, and the steps in (39)-(44) are still the same. Thus Theorem 2 is proven.

\section{APPENDIX C \\ SINR-MSE RELATION}

To prove the MSE duality, we first rewrite $\operatorname{SINR}_{i}^{M}$ in (32) as

$$
\begin{aligned}
\operatorname{SINR}_{i}^{M} & =\frac{p_{i}\left|\mathbf{u}_{i}^{H} \bigotimes_{l=1}^{L-1}\left(\tilde{\mathbf{H}}_{l}^{H} \mathbf{F}_{l}^{H}\right) \tilde{\mathbf{H}}_{L}^{H} \mathbf{v}_{i}\right|^{2}}{\mathbf{u}_{i}^{H} \mathbf{P}_{i} \mathbf{u}_{i}} \\
& \leq p_{i} \mathbf{v}_{i}^{H} \tilde{\mathbf{H}}_{L} \bigotimes_{l=L-1}^{1}\left(\mathbf{F}_{l} \tilde{\mathbf{H}}_{l}\right) \mathbf{P}_{i}^{-1} \bigotimes_{l=1}^{L-1}\left(\tilde{\mathbf{H}}_{l}^{H} \mathbf{F}_{l}^{H}\right) \tilde{\mathbf{H}}_{L}^{H} \mathbf{v}_{i}
\end{aligned}
$$

where $\mathbf{M}_{i}$ is defined in (33), and the inequality comes from Cauchy-Schwarz's inequality [34]. The upper bound is achieved by

$$
\mathbf{u}_{i}^{\mathrm{SINR}}=\alpha_{i} \mathbf{M}_{i}^{-1} \bigotimes_{l=1}^{L-1}\left(\tilde{\mathbf{H}}_{l}^{H} \mathbf{F}_{l}^{H}\right) \tilde{\mathbf{H}}_{L}^{H} \mathbf{v}_{i}
$$

Here $\alpha_{i} \neq 0$ is an arbitrary scalar.

Using (11), we can express the MSE of the $i$ th data stream for the MAC channel as ${ }^{7}$

$$
\begin{aligned}
& \mathrm{MSE}_{i}^{M}=\mathbb{E}\left[\left|\hat{s}_{i}^{M}-s_{i}^{M}\right|^{2}\right] \\
& =\mathbb{E}\left[p_{i}\left|\mathbf{u}_{i}^{H} \bigotimes_{l=1}^{L-1}\left(\mathbf{H}_{l}^{H} \mathbf{F}_{l}^{H}\right) \mathbf{H}_{L}^{H} \mathbf{v}_{i}\right|^{2}\right]+\mathbf{u}_{i}^{H} \mathbf{u}_{i} \\
& +\sum_{j=1, j \neq i}^{N_{b}} \mathbb{E}\left[p_{j}\left|\mathbf{u}_{i}^{H} \bigotimes_{l=1}^{L-1}\left(\mathbf{H}_{l}^{H} \mathbf{F}_{l}^{H}\right) \mathbf{H}_{L}^{H} \mathbf{v}_{j}\right|^{2}\right] \\
& +\sum_{k=1}^{L-1} \mathbb{E}\left[\mathbf{u}_{i}^{H} \bigotimes_{m=1}^{k}\left(\mathbf{H}_{m}^{H} \mathbf{F}_{m}^{H}\right) \bigotimes_{m=k}^{1}\left(\mathbf{F}_{m} \mathbf{H}_{m}\right) \mathbf{u}_{i}\right] \\
& -2 p_{i}^{\frac{1}{2}} \Re\left\{\mathbb{E}\left[\mathbf{u}_{i}^{H} \bigotimes_{l=1}^{L-1}\left(\mathbf{H}_{l}^{H} \mathbf{F}_{l}^{H}\right) \mathbf{H}_{L}^{H} \mathbf{v}_{i}\right]\right\}+1 .
\end{aligned}
$$

Using $\mathbf{M}_{i}$ defined in (33), (52) can be written as

$$
\begin{aligned}
\operatorname{MSE}_{i}^{M}= & p_{i}\left|\mathbf{u}_{i}^{H} \bigotimes_{l=1}^{L-1}\left(\tilde{\mathbf{H}}_{l}^{H} \mathbf{F}_{l}^{H}\right) \tilde{\mathbf{H}}_{L}^{H} \mathbf{v}_{i}\right|^{2}+\mathbf{u}_{i}^{H} \mathbf{M}_{i} \mathbf{u}_{i} \\
& -2 p_{i}^{\frac{1}{2}} \Re\left\{\mathbf{u}_{i}^{H} \bigotimes_{l=1}^{L-1}\left(\tilde{\mathbf{H}}_{l}^{H} \mathbf{F}_{l}^{H}\right) \tilde{\mathbf{H}}_{L}^{H} \mathbf{v}_{i}\right\}+1 .
\end{aligned}
$$

The optimal receive vector $\mathbf{u}_{i}$ minimizing (53) is the Wiener filter [35] and given by

$$
\begin{aligned}
\mathbf{u}_{i}^{\mathrm{MSE}} & =\left(\mathbf{M}_{i}+\tilde{\mathbf{v}}_{i} \tilde{\mathbf{v}}_{i}^{H}\right)^{-1} \tilde{\mathbf{v}}_{i} \\
& =\frac{\mathbf{M}_{i}^{-1} \tilde{\mathbf{v}}_{i}}{1+\tilde{\mathbf{v}}_{i}^{H} \mathbf{M}_{i}^{-1} \tilde{\mathbf{v}}_{i}}
\end{aligned}
$$

where $\tilde{\mathbf{v}}_{i} \triangleq p_{i}^{\frac{1}{2}} \bigotimes_{l=1}^{L-1}\left(\tilde{\mathbf{H}}_{l}^{H} \mathbf{F}_{l}^{H}\right) \tilde{\mathbf{H}}_{L}^{H} \mathbf{v}_{i}$ and the matrix inversion lemma, which is given by $(\mathbf{A}+\mathbf{B C D})^{-1}=\mathbf{A}^{-1}-$

${ }^{7}$ Since we consider single-user downlink and uplink multihop MIMO relay system in our proof, the inter-user interference term from (11) can be eliminated. In other words, the inter-user interference becomes intra-user interference when we treat multiple users as a "super" node as in Section IV. 
$\mathbf{A}^{-1} \mathbf{B}\left(\mathbf{D A}{ }^{-1} \mathbf{B}+\mathbf{C}^{-1}\right)^{-1} \mathbf{D} \mathbf{A}^{-1}$, is applied to obtain the second equation in (54).

Comparing (51) with (54), we find that $\mathbf{u}_{i}^{\mathrm{MSE}}$ in (54) also maximizes the SINR with $\alpha_{i}=p_{i}^{\frac{1}{2}} /\left(1+\tilde{\mathbf{v}}_{i}^{H} \mathbf{M}_{i}^{-1} \tilde{\mathbf{v}}_{i}\right)$. By substituting (54) back into (53), the MSE of the $i$ th data stream of the MAC system is given by

$$
\begin{aligned}
\operatorname{MSE}_{i}^{M} & =\frac{1}{1+\tilde{\mathbf{v}}_{i}^{H} \mathbf{M}_{i}^{-1} \tilde{\mathbf{v}}_{i}} \\
& =\frac{1}{1+\operatorname{SINR}_{i}^{M}} .
\end{aligned}
$$

\section{REFERENCES}

[1] P. Viswanath and D. Tse, "Sum capacity of the vector Gaussian broadcast channel and uplink-downlink duality," IEEE Trans. Inf. Theory, vol. 49, pp. 1912-1921, Aug. 2003.

[2] M. Schubert and H. Boche, "Solution of the multiuser downlink beamforming problem with individual SINR constraints," IEEE Trans. Veh. Technol., vol. 53, pp. 18-28, Jan. 2004.

[3] D. Tse and P. Viswanath, "On the capacity of the multiple antenna broadcast channel," in Proc. Multiantenna Channels: Capacity, Coding and Signal Process., DIMACS Workshop Amer. Math. Soc. 2003, Oct. 2002, vol. 62, pp. 87-105.

[4] A. Khachan, A. J. Tenenbaum, and R. Adve, "Joint transmitter-receiver optimization for downlink multiuser MIMO communications," IEEE Trans. Wireless Commun., 2006, submitted for publication.

[5] K. S. Gomadam and S. A. Jafar, "Duality of MIMO multiple access channel and broadcast channel with amplify-and-forward relays," IEEE Trans. Commun., vol. 58, pp. 211-217, Jan. 2010.

[6] Y. Rong and M. R. A. Khandaker, "On uplink-downlink duality of multihop MIMO relay channel," IEEE Trans. Wireless Commun., vol. 10, pp. 1923-1931, Jun. 2011.

[7] A. Liu, V. K. N. Lau, and Y. Liu, "Duality and optimization for generalized multi-hop MIMO amplify-and-forward relay networks with linear constraints," IEEE Trans. Signal Process., vol. 61, no. 9, pp. 23562365, May 2013.

[8] M. Ding and S. D. Blostein, "Uplink-downlink duality in normalized MSE or SINR under imperfect channel knowledge," in Proc. IEEE Global Telecommun. Conf., Washington, DC, Nov. 26-30, pp. 37863790, 2007.

[9] S. Shi and M. Schubert, "MMSE transmit optimization for multi-user multi-antenna systems," in Proc. IEEE Int. Conf. Acoustics, Speech, and Signal Process., Philadelphia, PA, Mar. 18-23, vol. 3, pp. 409-412, 2005.

[10] S. Shi, M. Schubert, and H. Boche, "Downlink MMSE transceiver optimization for multiuser MIMO systems: Duality and sum-MSE minimization," IEEE Trans. Signal Process., vol. 55, pp. 5436-5446, Nov. 2007.

[11] R. Hunger, M. Joham, and W. Utschick, "On the MSE-duality of the broadcast channel and the multiple access channel," IEEE. Trans. Signal Process., vol. 57, pp. 698-713, Feb. 2009.

[12] M. B. Shenouda and T. Davidson, "On the design of linear transceivers for multiuser systems with channel uncertainty," IEEE J. Sel. Areas Commun., vol. 26, pp. 1015-1024, Aug. 2008.

[13] P. Ubaidulla and A. Chockalingam, "Robust joint precoder/receive filter designs for multiuser MIMO downlink," in Proc. 10th IEEE Workshop Signal Process. Advances Wireless Commun., pp. 136-140, Jun. 2009.

[14] M. Ding and S. D. Blostein, "Relation between joint optimizations for multiuser MIMO uplink and downlink with imperfect CSI," in Proc. IEEE Int. Conf. Acoustics, Speech, Signal Process., Las Vegas, NV, pp. 3149-3152, Apr. 2008.

[15] T. Endeshaw, B. Chalise, and L. Vandendorpe, "MSE uplink-downlink duality of MIMO systems under imperfect CSI,", in 3rd IEEE International Workshop on Computational Advances in Multi-Sensor Adaptive Processing (CAMSAP), Aruba, 1316 Dec. 2009, pp. 384387.

[16] T. E. Bogale, B. K. Chalise, and L. Vandendorpe, "Robust transceiver optimization for downlink multiuser MIMO systems", IEEE Trans. Signal Process., vol. 59, pp. 446-453, Jan. 2011.

[17] J. Liu and Z. Qiu, "Sum MSE uplink-downlink duality of multiuser amplify-and-forward MIMO relay systems," IEEE Veh. Technol. Conf., San Francisco, CA, Sep. 5-8, 2011.

[18] N. Jindal, S. Vishwanath, and A. Goldsmith, "On the duality of Gaussian multiple-access and broadcast channels, IEEE Trans. Inf. Theory, vol. 50, no. 5, pp. 768-783, May 2004.
[19] S. Vishwanath, N. Jindal, and A. J. Goldsmith, "Duality, achievable rates, and sum-rate capacity of Gaussian MIMO broadcast channels," IEEE Trans. Inf. Theory, vol. 49, pp. 2658-2668, Oct. 2003.

[20] H. Weingarten, Y. Steinberg, and S. S. Shamai, "The capacity region of the Gaussian multiple-input multiple-output broadcast channel," IEEE Trans. Inf. Theory, vol. 52, no. 9, pp. 3936-3964, 2006.

[21] S. A. Jafar, K. S. Gomadam, and C. Huang, "Duality and rate optimization for multiple access and broadcast channels with amplify-andforward relays," IEEE Trans. Inf. Theory, vol. 53, pp. 3350-3370, Oct. 2007.

[22] S. Han, S. Ahn, E. Oh, and D. Hong, "Effect of channel estimation error on BER performance in cooperative transmission," IEEE Trans. Veh. Technol., vol. 58, pp. 2083-2088, May 2009.

[23] O. Amin, S. S. Ikki, and M. Uysal, "On the performance analysis of multirelay cooperative diversity systems with channel estimation errors," IEEE Trans. Veh. Technol., vol. 60, pp. 2050-2059, June 2011.

[24] A. S. Ibrahim and K. J. R. Liu, "Mitigating channel estimation error via cooperative communications," in Proc. IEEE ICC, 2009.

[25] S. S. Ikki and S. Aïssa, "Impact of imperfect channel estimation and co-channel interference on dual-hop relaying systems," IEEE Commun. Lett., vol. 16, pp. 324-327, Mar. 2012.

[26] M. Ding and S. D. Blostein, "MIMO minimum total MSE transceiver design with imperfect CSI at both ends," IEEE Trans. Signal Process., vol. 57, pp. 1141-1150, Mar. 2009.

[27] T. Yoo, E. Yoon, and A. Goldsmith, "MIMO capacity with channel uncertainty: Does feedback help?" in Proc. IEEE Global Telecommun. Conf., Dallas, TX, vol. 1, pp. 96-100, Nov. 29-Dec. 3, 2004.

[28] Y. Rong, "Simplified algorithms for optimizing multiuser multi-hop MIMO relay systems," IEEE Trans. Commun., vol. 59, pp. 2896-2904, Oct. 2011.

[29] Y. Rong, "Optimal linear nonregenerative multihop MIMO relays with MMSE-DFE receiver at the destination," IEEE Trans. Wireless Commun., vol. 9, pp. 2268-2279, Jul. 2010.

[30] Y. Rong, X. Tang, and Y. Hua, "A unified framework for optimizing linear non-regenerative multicarrier MIMO relay communication systems,'IEEE Trans. Signal Process., vol. 57, pp. 4837-4851, Dec. 2009.

[31] M. R. A. Khandaker and Y. Rong, "Joint transceiver optimization for multiuser MIMO relay communication systems," IEEE Trans. Signal Process., vol. 60, pp. 5977-5986, Nov. 2012.

[32] B. Hassibi and B. M. Hochwald, "How much training is needed in multiple-antenna wireless link?," IEEE Trans. Inf. Theory, vol. 49, no. 4, pp. 951-963, Apr. 2003.

[33] L. Zhang, R. Zhang, Y.-C. Liang, Y. Xin, and H. V. Poor, "On Gaussian MIMO BC-MAC duality with multiple transmit covariance constraints," IEEE Trans. Inf. Theory, vol. 58, no. 4, pp. 2064-2078, Apr. 2012.

[34] D. Palomar, J. Cioffi, and M. Lagunas, "Joint Tx-Rx beamforming design for multicarrier MIMO channels: A unified framework for convex optimization," IEEE Trans. Signal Process., vol. 51, no. 9, pp. 23812401, Sep. 2003.

[35] S. M. Kay, Fundamentals of Statistical Signal Processing: Estimation Theory. Englewood Cliffs, NJ: Prentice-Hall, 1993.

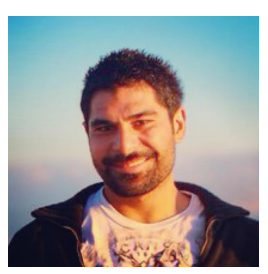

Ali Cagatay Cirik received the B.S and M.S. degrees in telecommunications and electronics engineering from Sabanci University, Istanbul, Turkey, in 2007 and 2009, respectively, and Ph.D. degree in electrical engineering from University of California, Riverside in 2014. Currently, he is working as a research scientist at Centre for Wireless Communications, University of Oulu, Finland.

His industry experience includes internships at Mitsubishi Electric Research Labs, Cambridge, MA, in 2012 and at Broadcom Corporation, Irvine, CA, in 2013. His primary research interests are full-duplex communication, MIMO signal processing, and convex optimization. 


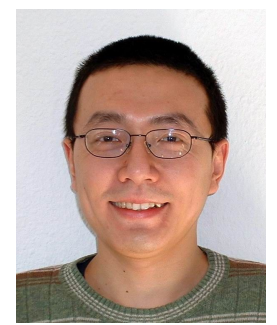

Yue Rong (S'03-M'06-SM'11) received the Ph.D. degree (summa cum laude) in electrical engineering from the Darmstadt University of Technology, Darmstadt, Germany, in 2005.

He was a Post-Doctoral Researcher with the Department of Electrical Engineering, University of California, Riverside, from February 2006 to November 2007. Since December 2007, he has been with the Department of Electrical and Computer Engineering, Curtin University, Bentley, Australia, where he is currently an Associate Professor. His research interests include signal processing for communications, wireless communications, underwater acoustic communications, applications of linear algebra and optimization methods, and statistical and array signal processing.

Dr. Rong was a recipient of the Best Paper Award at the 2011 International Conference on Wireless Communications and Signal Processing, the Best Paper Award at the 2010 Asia-Pacific Conference on Communications, and the Young Researcher of the Year Award of the Faculty of Science and Engineering at Curtin University in 2010. He is an Editor of the IEEE WIRELESS COMMUNICATIONS LeTTERS, a Guest Editor of the IEEE JOURNAL on Selected AREas in Communications special issue on theories and methods for advanced wireless relays, and was a TPC Member for the IEEE ICC, WCSP, IWCMC, and ChinaCom.

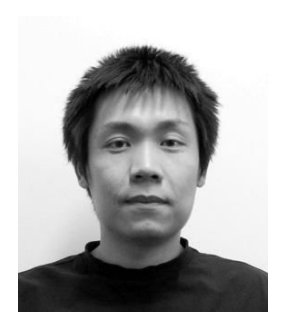

Yiming $\mathrm{Ma}$ is a hardworking $\mathrm{PhD}$ candidate and looking forward to graduating. He received the B.E. degree of Electrical Engineering from China University of Mining and Technology, Xuzhou, Jiangsu, China, the M.S. degree of technology from Western Carolina University, Cullowhee, North Carolina, USA, and the M.S. degree of electrical engineering from University of California, Riverside, USA, in 2008, 2010 and 2011, respectively. He is currently working towards the Ph.D. degree in electrical engineering at University of California, Riverside. His research interests include wireless communications and multiuser multicarrier optimization.

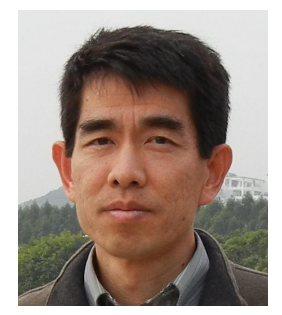

Yingbo Hua (S'86-M'88-SM'92-F'02) received a B.S. degree (1982) from Southeast University, Nanjing, China, a M.S. degree (1983) and a Ph.D. degree (1988) from Syracuse University, Syracuse, NY. He was a Lecturer (1990-1992), a Senior Lecturer (1993-1995), and a Reader and Associate Professor (1996-2000) with the University of Melbourne, Australia. He was a Visiting Faculty Member with Hong Kong University of Science and Technology (19992000), and a Consultant with Microsoft Research, WA (summer 2000). Since 2001, he has been with the University of California at Riverside, where he is a Senior Full Professor. Dr. Hua has served as Editor, Guest Editor, Member of Editorial Board and/or Member of Steering Committee for IEEE Transactions on Signal Processing, IEEE Signal Processing Letters, EURASIP Signal Processing, IEEE Signal Processing Magazine, IEEE Journal of Selected Areas in Communications, and IEEE Wireless Communication Letters. He has been a Member of IEEE Signal Processing Society's Technical Committees for Underwater Acoustic Signal Processing, Sensor Array and Multichannel Signal Processing, and Signal Processing for Communication and Networking. He has served as member of Technical and/or Advisory Committees for over forty international conferences and workshops. He has authored over three hundreds of articles and coedited three volumes of books, with more than six thousands of citations, in the fields of Sensing, Signal Processing and Communications. $\mathrm{He}$ is a Fellow of IEEE and AAAS. 